Distribution

Category UC-406

SAND94-2423

Unlimited Release

Printed January 1995

\title{
TIME-OPTIMAL CONTROL OF THE MAGNETICALLY LEVITATED PHOTOLITHOGRAPHY PLATEN
}

\author{
Jim Redmond \\ Structural Dynamics and Vibration Control Department 1434 \\ Susan Tucker \\ Manufacturing Control Subsystems Department 2338
}

Sandia National Laboratories

Albuquerque, NM 87185-0501

\begin{abstract}
This report summarizes two approaches to time-optimal control of a nonlinear magnetically levitated platen. The system of interest is a candidate technology for nextgeneration photolithography machines used in the manufacture of integrated circuits. The dynamics and the variable peak control force of the electro-magnetic actuators preclude the direct application of classical time-optimal control methodologies for determining optimal rest-to-rest maneuver strategies. Therefore, this study explores alternate approaches using a previously developed computer simulation. In the first approach, conservative estimates of the available control forces are used to generate suboptimal switching curves. In the second approach, exact solutions are determined iteratively and used as a training set for an artificial neural network. The trained network provides optimal actuator switching times that incorporate the full nonlinearities of the magnetic levitation actuators. Sample problems illustrate the effectiveness of these techniques as compared to traditional proportional-derivative control.




\section{ACKNOWLEDGEMENTS}

The authors are indebted to Tony Smith, Stew Kohler, and Suzanne Stanton of Department 2338 for initiating and supporting this project and for supplying the computer model of the magnetically levitated fine stage. 


\section{DISCLAIMER}

This report was prepared as an account of work sponsored by an agency of the United States Government. Neither the United States Government nor any agency thereof, nor any of their employees, make any warranty, express or implied, or assumes any legal liability or responsibility for the accuracy, completeness, or usefulness of any information, apparatus, product, or process disclosed, or represents that its use would not infringe privately owned rights. Reference herein to any specific commercial product, process, or service by trade name, trademark, manufacturer, or otherwise does not necessarily constitute or imply its endorsement, recommendation, or favoring by the United States Government or any agency thereof. The views and opinions of authors expressed herein do not necessarily state or reflect those of the United. States Government or any agency thereof. 



\section{DISCLAIMER}

Portions of this document may be illegible in electronic image products. Images are produced from the best available original document. 


\section{TABLE OF CONTENTS}





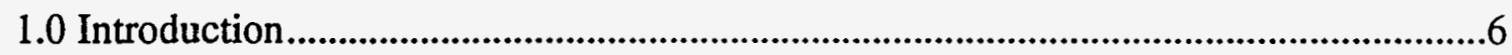

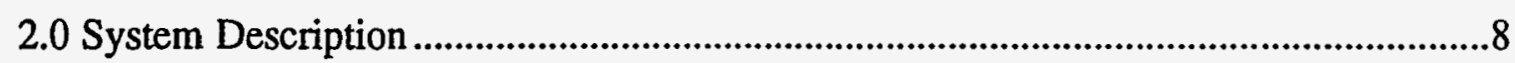

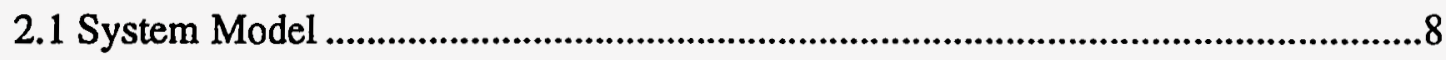

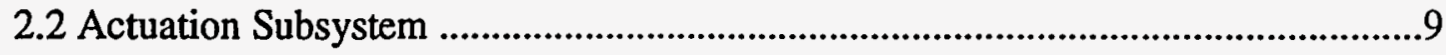

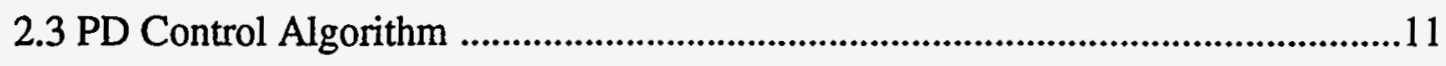

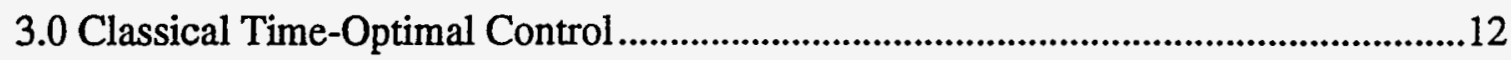

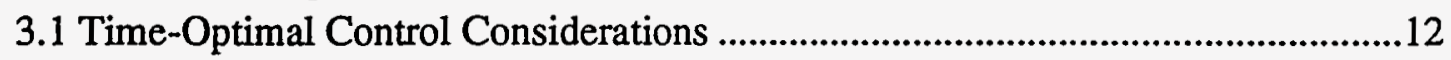

3.2 Classical Rigid Body Time-Optimal Control ..................................................12

3.3 Conservative Control Force Estimates.............................................................13

3.4 Customizing for Actuator Dynamics ........................................................14

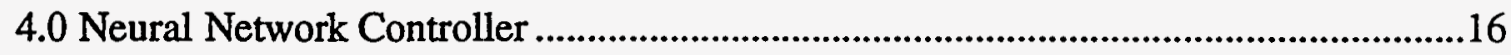

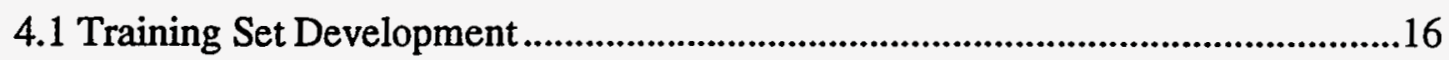

4.1.1 Time-Optimal Maneuver Strategy ....................................................16

4.1.2 Determination of Training Points .........................................................17

4.1.3 Sample Training Point Determination .................................................18

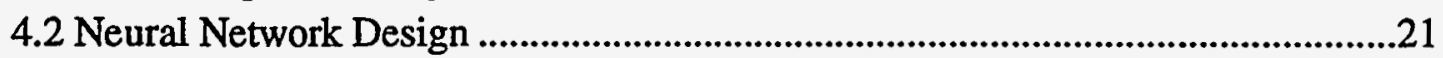

4.2.1 General Regression Neural Networks................................................21

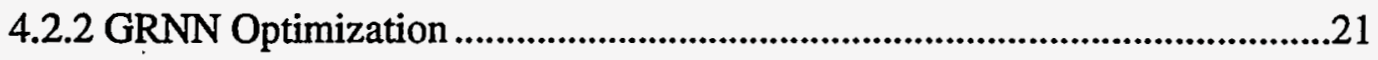

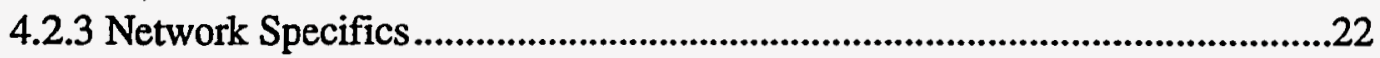

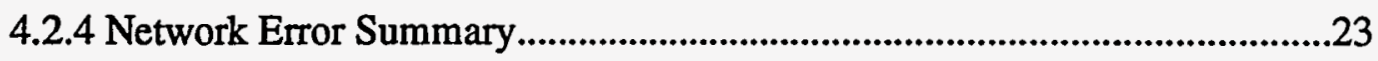

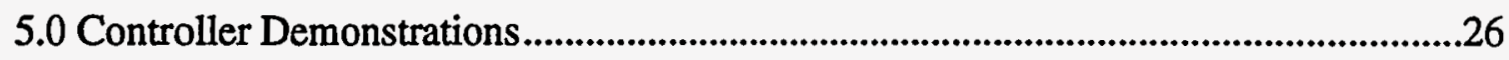

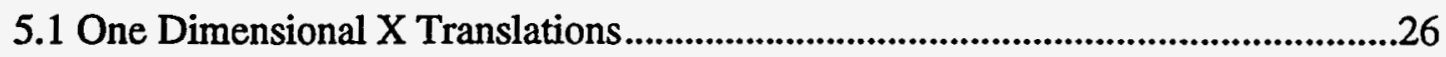

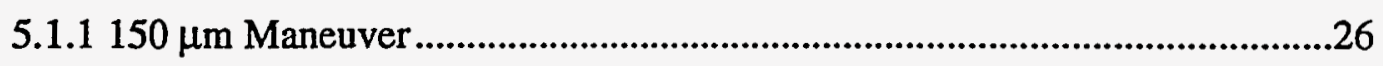

$5.1 .250 \mu \mathrm{m}$ Maneuver......................................................................................28

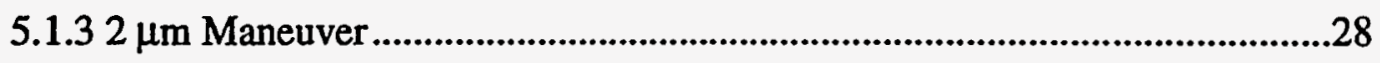

5.1.4 Average Performance......................................................................29

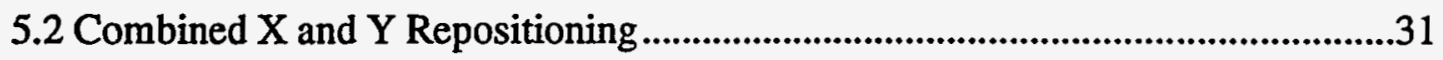

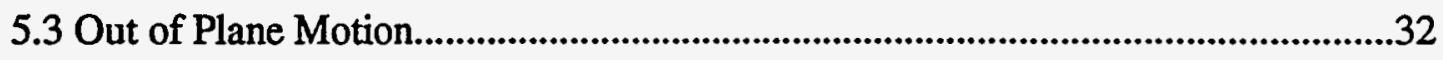

6.0 Recommendations for Future Work.........................................................................35

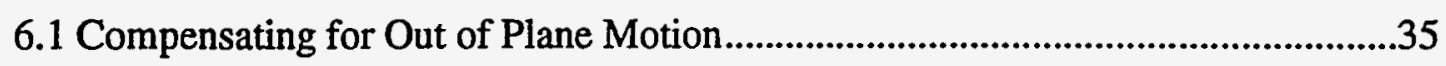

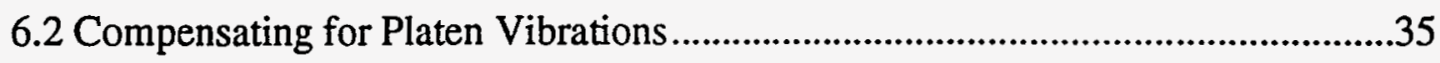



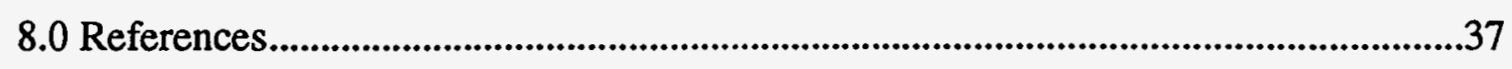




\section{LIST OF FIGURES}

1. Schematic Diagram of Fine Stage Platen...................................................................

2. Classical Time-Optimal Control Regions. ...............................................................14

3. Triangulation to Determine Second Switching Time..............................................18

4. Convergence to Optimal Velocity Profile.....................................................................19

5. Convergence to Optimal Force Profile ......................................................................19

6. Convergence to Optimal Displacement Profile...............................................................

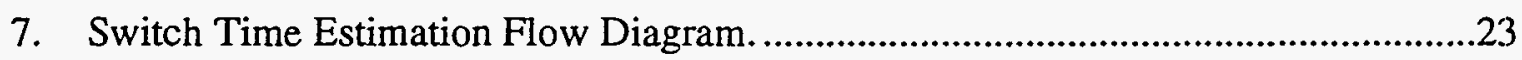

8. $\mathrm{T}_{\mathrm{sl}}$ Error Bounds as a Function of Movesize..........................................................24

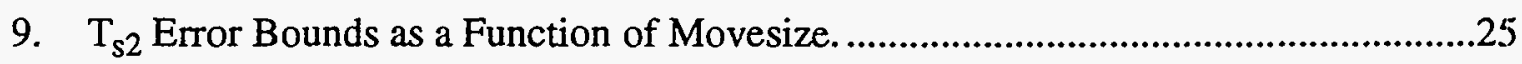

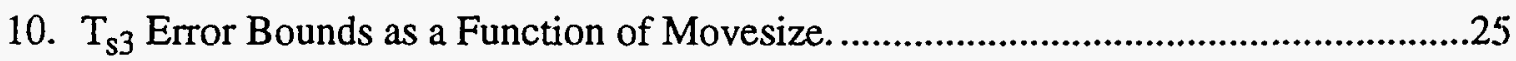

11. X Modal Displacement History for $150 \mu \mathrm{m}$ Maneuver....................................................

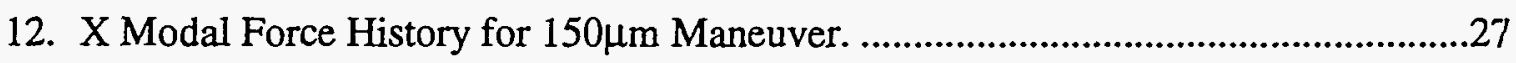

13. X Modal Displacement History for $50 \mu \mathrm{m}$ Maneuver....................................................28

14. X Modal Force History for $50 \mu \mathrm{m}$ Maneuver. ………..................................................29

15. X Modal Displacement History for $2 \mu \mathrm{m}$ Maneuver.....................................................29

16. X Modal Force History for $2 \mu \mathrm{m}$ Maneuver. .........................................................30

17. Comparison of Controller Performances for One Dimensional Maneuvers. ...............31

18. Two Dimensional Maneuver Displacement Histories..............................................32

19. X Axis Rotation for Two Dimensional Maneuver. .......................................................33

20. Y Axis Rotation for Two Dimensional Maneuver. ..................................................33 


\section{LIST OF TABLES}

1. Photolithography Fine Stage Parameters......................................................................10

2. Sample Neural Network Training Patterns Using a 100kHz Clock. ..........................20

3. Network Training Data Summary................................................................................23 


\subsection{INTRODUCTION}

Recent advances in control systems technology have focused on the development of smart materials and their use in active shape control (Ref. 1-3). Although these materials are well suited for damping a structure's flexible body modes, they provide no direct control authority over a structure's rigid body motion. Consequently, applications that require rigid body maneuvers must employ alternative sources of actuation. For applications requiring control of a primary structure relative to a structural frame, electromagnetic actuation is gaining in popularity because it provides precision control authority with minimal frictional losses (Ref. 4). Recently, this method of actuation was employed to improve the process of integrated circuit (IC) manufacturing (Ref. 5). Based on advanced photolithography techniques, IC manufacturing requires precision placement of a platen supporting a silicon wafer. With magnetic levitation actuators, accuracy of better than 10 $\mathrm{nm}$ has been achieved for typical rest-to-rest maneuvers of the platen. As with other actuation devices, the use of time-optimal control strategies would improve the speed of the manufacturing process. However, inherent nonlinearities of magnetic levitation devices preclude generating traditional time-optimal switching surfaces.

In this report, two approaches to developing time-optimal control strategies for the platen are presented. First, an approximate time-optimal feedback strategy is developed by using linearized estimates of the available control forces to generate simple modal switching curves (Ref. 6). Although this technique proves to be useful for relatively large maneuvers, substantial performance gains can be realized by incorporating the actuator nonlinearities and maximizing the control input levels. In the second approach, an artificial neural network is trained to provide near optimal switching times for conducting one dimensional rest-to-rest maneuvers. Although they have been successfully applied to a variety of controls problems, artificial neural networks are especially useful for identification and control of nonlinear systems (Ref. 7). Their application to time-optimal control problems are becoming more prevalent (Ref. 8), however no known application exists for systems that include magnetic levitation actuators.

The motion of the platen and wafer containing the ICs is governed by a two stage hierarchial position control scheme. The first (coarse) stage executes large scale two dimensional translations via motor driven screws. The second (fine) stage governs six degree of freedom rigid body motion via electro-magnetic actuators. This paper focuses on the optimization of the fine stage control system for conducting two dimensional translational maneuvers. The development is based on a computer simulation that has been shown to accurately model the behavior of the actual hardware (Ref. 9).

A brief description of the system is given in the following section. In section 3, the inherent nonlinearities of the magnetic levitation actuators are circumvented and an approximate time-optimal control strategy is developed based on the classical solution for rigid bodies. This controller drives the platen to the vicinity of the target destination where a conventional proportional-derivative (PD) controller assumes control authority and completes the maneuver. This transition to PD control is required to correct for trajectory errors that can result from external disturbances and imprecise sampling of the phase space. In section 4 an open-loop neural network controller is developed that incorporates the actuator nonlinearities. Again, control authority is transferred to a PD controller near the terminal point to accommodate possible external disturbances and network errors. Both 
time-optimal controllers are demonstrated on the computer simulation in section 5 and the performance is compared to the performance obtained using only PD control. Substantial reductions in settling time are demonstrated using the time-optimal strategies over a broad range of motion. However, the use of the time-optimal controllers is accompanied by inadvertent out of plane motion. This motion and the possibility of instability resulting from the platen flexibility form the basis for our recommendations for further work presented in section 6. Finally, some concluding remarks are given in section 7. 


\subsection{SYSTEM DESCRIPTION}

A complete description of the magnetically levitated fine stage is given in Reference 5. However, the details pertinent to this study are restated here for clarity. First, the mathematical model of the six degree of freedom rigid body dynamics is presented under the assumption of small angular motion and $\mathrm{Z}$ axis symmetry. Then, the pairing of the sixteen uni-directional actuators into eight bi-directional units is summarized, with a simple transformation relating actuator pair forces to modal forces. Finally, the analog version of the original PD control algorithm is presented. A remnant of this controller is maintained for both of the time-optimal controllers.

\subsection{System Model}

A schematic diagram of the fine stage platen is shown in Figure 1. The inertial coordinate system XYZ is nominally located at the platen center of mass when the platen is centered in the fixed coarse stage. The chuck containing the ICs has been omitted for clarity. The fine stage floats within the coarse stage frame which contains the actuation hardware. Three permanent magnets are located below the platen to offset the effects of gravity, alleviating the demands placed on the actuators. Translational motions along the $\mathrm{X}$ and $Y$ axes are limited to $+/-250 \mu \mathrm{m}$ beyond which the platen edges contact the coarse stage frame. The platen is composed primarily of aluminum and is assumed to be rigid for this study. For small angles, the angular velocities of the body in inertial coordinates are equal to the rate of change of the orientation angles, and the dynamics are described by

$$
\begin{aligned}
& m \ddot{x}(t)=F_{x}(t) \\
& m \ddot{y}(t)=F_{y}(t)
\end{aligned}
$$

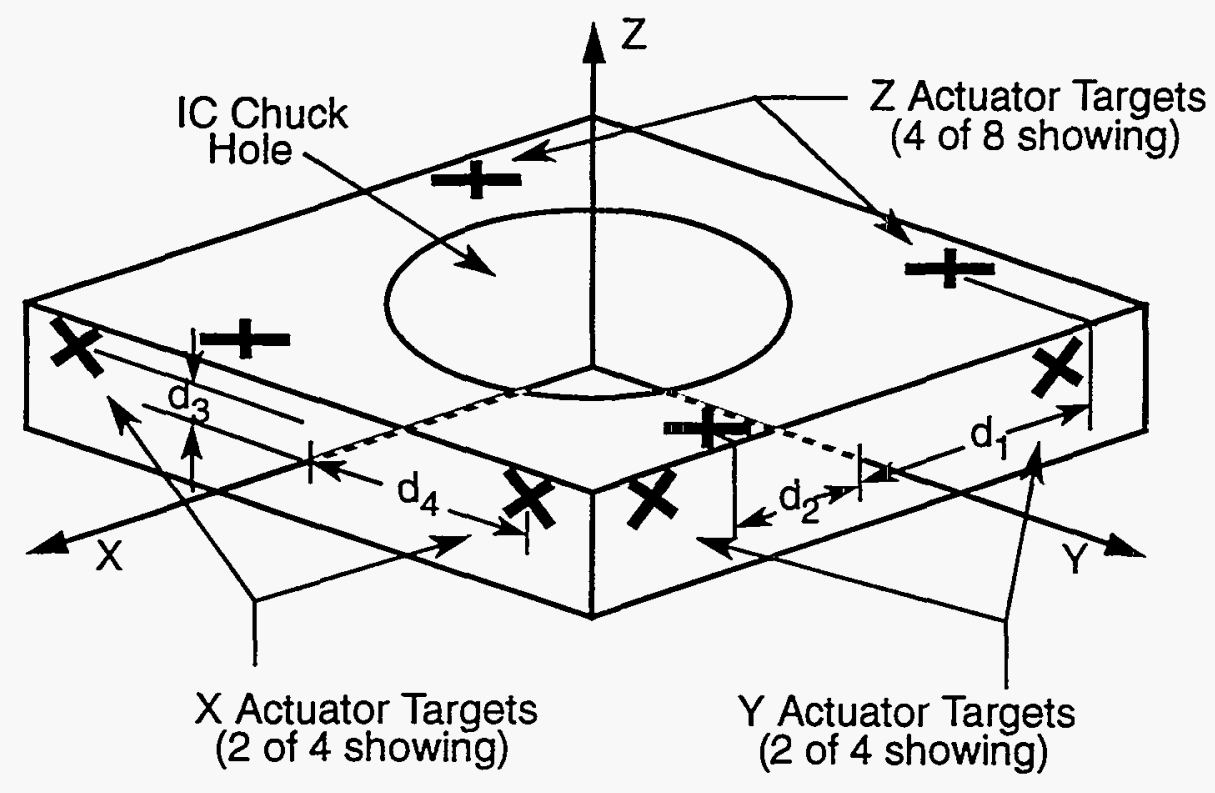

Figure 1. - Schematic Diagram of Fine Stage Platen. 


$$
\begin{gathered}
m \ddot{z}(t)=F_{z}(t) \\
I_{x} \ddot{\theta}_{x}(t)+\left(I_{z}-I_{y}\right) \ddot{\theta}_{y}(t) \dot{\theta}_{z}(t)=T_{x}(t) \\
I_{y} \ddot{\theta}_{y}(t)+\left(I_{x}-I_{z}\right) \dot{\theta}_{x}(t) \dot{\theta}_{z}(t)=T_{y}(t) \\
I_{z} \ddot{\theta}_{z}(t)=T_{z}(t)
\end{gathered}
$$

in which $m$ is the platen mass, $I_{x}, I_{y}, I_{z}$ are the principal mass moments of inertia, $x(t)$, $y(t), z(t), \theta_{x}(t), \theta_{y}(t), \theta_{z}(t)$ are the modal (rigid body) displacements and rotations, and $F_{x}(t), F_{y}(t), F_{z}(t), T_{x}(t), T_{y}(t), T_{z}(t)$ are the modal control forces and torques. The modal displacements are extracted via a geometric transformation of the signals from six capacitive displacement sensors located in the platen frame. The sensor measurements are processed in a digital signal processor with a sampling rate of $2500 \mathrm{~Hz}$. As shown in equation If, the decoupling of the $Z$ axis rotation is a consequence of the symmetry displayed in Figure 1. Although they were not considered in the design of the PD control because their effect was found to be negligible over the range of motion, the crossaxis coupling of the $X$ and $Y$ axes rotations shown in equations $1 d$ and $1 e$ are included in the simulation for additional accuracy. Note that no damping terms are included in equation 1 since the magnetic levitation minimizes frictional losses.

\subsection{Actuation Subsystem}

For this study, the primary role of the fine stage is assumed to be one of conducting rest-to-rest $\mathrm{X}$ and $\mathrm{Y}$ translational maneuvers while regulating the other rigid body modes. This fine stage, which provides positioning accuracies better than $10 \mathrm{~nm}$, is activated after the coarse stage directs gross $X$ and $Y$ translations via motor driven ball screws. The actual range of motion required of the fine stage depends on the accuracy of the coarse stage which is nominally $+/-100 \mu \mathrm{m}$. Sixteen magnetic levitation actuators whose outputs are approximately proportional to the square of the supply currents and inversely proportional to the square of the actuator gaps provide the forces for controlling fine stage motion. Since each magnetic levitation device is capable of producing only uni-directional pulling forces, the sixteen devices are grouped into eight bi-directional actuator pairs. The actuator forces are related to the modal forces via the geometric transformation

$$
F(t)=D F_{a}(t)
$$

The transformation matrix, the modal force vector, and the actuator force vector are defined as 


$$
\begin{gathered}
D=\left[\begin{array}{cccccccc}
1 & 1 & 0 & 0 & 0 & 0 & 0 & 0 \\
0 & 0 & 1 & 1 & 0 & 0 & 0 & 0 \\
0 & 0 & 0 & 0 & 1 & 1 & 1 & 1 \\
0 & 0 & d_{3} & d_{3} & -c & c & c & -c \\
-d_{3} & -d_{3} & 0 & 0 & c & -c & c & -c \\
d_{4} & -d_{4} & d_{4} & -d_{4} & 0 & 0 & 0 & 0
\end{array}\right] \\
F(t)=\left[\begin{array}{llllll}
F_{x}(t) & F_{y}(t) & F_{z}(t) & T_{x}(t) & T_{y}(t) & T_{z}(t)
\end{array}\right]^{T} \\
F_{a}(t)=\left[\begin{array}{llll}
F_{a 1}(t) & F_{a 2}(t) & \ldots & F_{a 8}(t)
\end{array}\right]^{T}
\end{gathered}
$$

in which $c=0.5\left(d_{1}+d_{2}\right), d_{1}, d_{2}, d_{3}, d_{4}$ represent actuator moment arms, and $F_{a i}(t), \mathrm{i}=1-8$ represent the forces supplied by the actuator pairs. The pertinent mass properties and moment arms are summarized in Table 1.

Table 1: Photolithography Fine Stage Parameters

\begin{tabular}{|c|c|}
\hline Platen Mass $(\mathrm{m})$ & 0.78 slugs \\
\hline X-Axis Mass Moment of Inertia $\left(\mathrm{I}_{\mathrm{x}}\right)$ & 8.4 slugs-in $^{2}$ \\
\hline Y-Axis Mass Moment of Inertia $\left(\mathrm{I}_{\mathrm{y}}\right)$ & 8.4 slugs-in $^{2}$ \\
\hline Z-Axis Mass Moment of Inertia $\left(\mathrm{I}_{\mathrm{z}}\right)$ & 15.6 slugs-in $^{2}$ \\
\hline Moment Arm d $\mathrm{d}_{1}$ & $5.145 \mathrm{in}$ \\
\hline Moment Arm d $\mathrm{d}_{2}$ & $2.712 \mathrm{in}$ \\
\hline Moment Arm $\mathrm{d}_{3}$ & $0.658 \mathrm{in}$ \\
\hline Moment Arm $\mathrm{d}_{4}$ & 4.75 in \\
\hline
\end{tabular}

Each actuator pair is nominally capable of generating $+/-15 \mathrm{lbs}(66.72 \mathrm{~N})$ of control force when the platen is centered in the coarse stage frame. However, this peak actuator force can vary from 10 to $25 \mathrm{lbs}(44.48$ to $111.2 \mathrm{~N}$ ) depending on the platen location and the sign of the actuator pair force. The desired forces from the actuator pairs are based on the desired modal forces from the control algorithm and are computed as the minimum norm solution to the under-determined system of equation 2. The actuator gaps and desired actuator forces specify the required supply current for each actuator pair. Since an explicit expression for the required supply current is difficult to obtain due to the actuator nonlinearities, a look-up table is used to designate the proper currents for large forces, while a curve fit is used for small forces (Ref. 5). The resulting actuator forces are transformed in 
the simulation to yield the actual modal forces used to drive the table motion. To be consistent with the hardware, a delay of $200 \mathrm{~ms}$ is included in the simulation to mimic the input to output computation time.

\subsection{PD Control Algorithm}

Currently, the hardware is equipped with a critically-damped PD controller which neglects the cross axis coupling illustrated in EQ $1 \mathrm{~d}-\mathrm{f}$. Defining the target point as the origin of the phase plane, this control law can be summarized in continuous time as

$$
F(t)=-2 \omega M \dot{X}(t)-\omega^{2} M X(t)
$$

in which $\omega$ is the controller bandwidth, and the inertia matrix and position vector are defined by

$$
\begin{aligned}
& M=\operatorname{diag}\left[m m m I_{x} I_{y} I_{z}\right] \\
& X(t)=\left[\begin{array}{llllll}
x(t) & y(t) & z(t) & \theta_{x}(t) & \theta_{y}(t) & \theta_{z}(t)
\end{array}\right]^{T}
\end{aligned}
$$

In this study, the derivative of the position vector is approximated using a simple three point backward difference approximation (Ref. 10)

$$
\dot{X}(t) \approx \frac{1}{2 T}[X(t-2 T)-4 X(t-T)+3 X(t)] .
$$

in which $T$ is the sampling period. For nominal translations, the bandwidth is limited to approximately $50 \mathrm{hz}$ to avoid actuator saturation throughout the full range of possible motion. Although temporary saturation is not harmful to the actuators, it can lead to an under-damped response and wasted chip processing time. This limitation prompted our investigation into the applicability of time-optimal control strategies. 


\subsection{CLASSICAL TIME-OPTIMAL CONTROL}

In this section, classical time-optimal phase plane switching logic is modified to accommodate the complexities of the electro-magnetic actuators. Conservative switch curves are generated by limiting the maximum available actuator forces, and a region of $P D$ control is maintained near the terminal point to correct for trajectory errors resulting from coarse sampling of the phase plane. The result is a hierarchial control law that governs the desired modal control forces based on the position of the platen in the state space.

\subsection{Time-Optimal Control Considerations}

Time-optimal control of low-order completely controllable systems is of the classical bang-bang form and can be readily obtained by minimizing the associated Hamiltonian and subsequently generating state based switching curves (Ref. 11). For highorder systems, numerical techniques exist for finding open-loop solutions for a given maneuver. However, several intricacies of the fine stage preclude the direct application of classical techniques. For example, the actuators are incapable of instantaneous switching between full positive and full negative forces as a consequence of the time delays associated with the finite sampling rate, processing operations, and actuator dynamics. Furthermore, the peak actuator forces are not constant but instead are complicated functions of the platen position and supply currents that are determined from look-up tables. Additionally, although each individual mode is completely controllable with respect to the modal control forces, the coupling of the modes through the actuator forces according to equation 3 renders the overall system singular in the control (Ref. 12). Therefore, the time-optimal control is not completely specified by the simple necessary conditions of optimality. Although higher order conditions can be considered to derive an optimal control strategy for singular systems, the variation of the peak control force as a function of platen position makes this approach impractical.

The approximate technique developed in this section is based on designing separate time-optimal control laws for each of the system's modes assuming a constant peak modal force level. Because each of these simplified modal time-optimal control problems are normal, classical bang-bang switching curves can be developed to govern the modal control forces. Afterward, some modifications to the basic switching curves are required to account for the significant processing delays and actuator dynamics. Since each of the translational modes in equation 1 have a similar form, the following development will concentrate on translations along the $\mathrm{X}$ axis. Application of the final switching strategy to the $\mathrm{Y}$ axis follows directly.

\subsection{Classical Rigid Body Time-Ontimal Control}

Assuming a constant peak modal force along the $\mathrm{X}$ axis, the classical time-optimal switch curve is given as a function of velocity as (Ref. 11)

$$
\bar{x}=\frac{-m}{2 F_{x}} \dot{x}|\dot{x}|
$$


where $\bar{x}$ is the modal switch curve displacement, $\dot{x}$ is the velocity, and $F_{x}$ is the nominally available peak modal control force which is obtained by summing the contributions from each of the pertinent actuators. The optimal feedback switching strategy is summarized as

$$
\begin{gathered}
x \neq \bar{x} \rightarrow F_{x}=F_{x} \operatorname{sgn}(\bar{x}-x) \\
x=\bar{x} \rightarrow F_{x}=-F_{x} \operatorname{sgn}(\dot{x})
\end{gathered}
$$

According to the above control law, the modal control force assumes a maximum level (t+ or -) until the modal trajectory intersects the switch curve. At that instant, the control executes a single sign switch and the phase trajectory follows the switch curve to the origin.

\subsection{Conservative Control Force Estimates}

The possibility of actuator saturation mandates a modification to the control of equation 8. For example, if the $\mathrm{X}$ translational and $\mathrm{Z}$ rotational modes simultaneously demand large control forces, then actuator $F_{a 1}$ will likely saturate. Consequently, the modal control forces required to satisfy the switching strategies are not attainable. This scenario may not often occur during operation since the role of the fine stage is primarily one of conducting X-Y maneuvers while regulating out of plane motion. However, actuator saturation will likely occur when the platen undergoes large displacements and the peak actuator forces diminish, eventually dropping below that required to satisfy the switch curve. Therefore, this possibility is included for completeness.

During the early phase of a rest-to-rest maneuver, actuator saturation is not harmful since no precise path is required to transfer the mode to the switch curve (Ref. 13). However, in the final phase of control, precise actuation is required for the mode to follow the switch curve to the origin. Any decrease in the modal force will cause the mode to overshoot its target and produce oscillatory motion. To prevent this, the switch curve must be designed based on a conservative estimate of the maximum modal control force that can be produced under usual circumstances. The modified switch curve becomes

$$
\bar{x}=\frac{-m}{2 \alpha \bar{F}_{x}} \dot{x}|\dot{x}|
$$

where $\alpha$ is a scaling factor used to adjust the peak modal force. With $\alpha$ assigned a value between 0 and 1 depending on the likelihood of saturation at some point during the transfer to the terminal point, the optimal switching strategy becomes

$$
\begin{aligned}
& x \neq \bar{x} \rightarrow F_{x}=\hat{F}_{x} \operatorname{sgn}(\bar{x}-x) \\
& x=\bar{x} \rightarrow F_{x}=-\alpha F_{x} \operatorname{sgn}(\dot{x})
\end{aligned}
$$






Figure 2. - Classical Time-Optimal Control Regions.

where $\hat{F}_{x}$ represents all force available regardless of switch curve design. In this modified control scheme, the modal control force assumes whatever force is available until the switch curve is reached. The control then switches sign and assumes the conservative control force level, with the modal trajectory following the path traced by the switch curve. Although some loss of performance results from conservatively estimating the available control force on the switch curve, the losses that can result from actuator saturation are generally greater.

\subsection{Customizing For Actuator Dynamics}

Before applying the switching algorithm to the fine stage positioning system, other modifications to the control strategy must be made to account for the time required by the controller to switch between peak positive and peak negative control forces. The elapsed time for transition is a function of the actuator dynamics and the timing of the sampling relative to the switch curve. Thus, an onset switch curve is defined to initiate the control transition prior to reaching the primary switch curve. Assuming that the control follows a half cosine profile in switching between peak positive and peak negative force, the onset switch curve can be expressed as (Ref. 14)

$$
\bar{x}_{o}=\frac{-m}{2 \alpha F_{x}} \dot{x}|\dot{x}|-T_{t} \dot{x}-\frac{2 T_{t}^{2} \alpha F_{x}}{m \pi^{2}} \operatorname{sgn}(\dot{x})
$$

where $\bar{x}_{o}$ is the switch onset displacement and $T_{t}$ is the time required for transition from peak to peak. The transition time must be selected such that the modal trajectory closely coincides with the primary switch curve when the control transition is complete. As shown in Figure 2, the region between the primary and the onset switch curves is denoted $S$. To accommodate the finite time required for the actuator force to make the transition from the conservative peak force to zero, a second transition time can be defined to shut off the 
actuators such that the system coasts to the terminal point, eventually coming to rest as the control force vanishes. However, since only limited resolution of the phase plane is available, this approach is impractical. Instead, a PD controller is used in region $R$ to complete the transition to the terminal point. This controller provides the added benefit of correcting for any discrepancies in the trajectory relative to the switch curve which result from modelling inaccuracies and an imprecise sampling of the phase plane. The left and right bounds of $R$ are determined by equating the conservative peak force with the PD control law to obtain

$$
\pm \alpha F_{x}=-2 m \omega \dot{x}-m \omega^{2} x
$$

The upper and lower bounds of the region are based on the points of intersection of the left and right bounds with the primary switch curve as shown in Figure 2. Setting $x$ in equation 12 equal to $\bar{x}$ in equation 9 and solving for the 6 points of intersection ( 3 for each of the right and left bounds), the upper and lower bounds of $R$ are given by the points closest to the origin as

$$
\dot{x}= \pm(2-\sqrt{2}) \frac{\alpha F_{x}}{m \omega}
$$

Defining the bounds of $R$ as in equation 13 ensures a smooth transition from time-optimal to PD control along the switch curve. Combining all of the modifications, the approximate time-optimal feedback strategy can be summarized in the following controller herein referred to as TOC

$$
\begin{gathered}
x \in R \rightarrow F_{x}=-2 m \omega \dot{x}-m \omega^{2} x \\
x \in S \rightarrow F_{x}=-\alpha F_{x} \operatorname{sgn}(\dot{x}) \\
x \notin \operatorname{Ror} S \rightarrow F_{x}=\hat{F}_{x} \operatorname{sgn}\left(\bar{x}_{o}-x\right)
\end{gathered}
$$

The fundamental difference between the approximate TOC strategy described in equation 14 and the original PD control strategy described in equation 4 is in the treatment of the actuator bounds. Instead of limiting controller bandwidth to avoid saturation, the time-optimal control uses saturated (or nearly saturated) actuator forces to drive the system to a region of PD control. Although the size of the region $R$ is dictated by controller bandwidth constraints, the limiting bandwidth is determined by the speed of the actuator dynamics, not by actuator saturation. 


\subsection{NEURAL NETWORK CONTROLLER}

The controller developed in this section uses an artificial neural network to produce optimal switching times that account for nonlinear actuator dynamics. Recall that the main purpose of the fine stage positioning system is to conduct $X$ and $Y$ translations in the range $+/-100 \mu \mathrm{m}$ while regulating the other rigid body modes. As such, the problem is simplified somewhat in that the control for the $\mathrm{X}$ and $\mathrm{Y}$ translations can be treated independently since these modes do not share common actuators as shown in equation 3 . Therefore, we will consider only rest-to-rest translations along the $\mathrm{X}$ axis beginning at point $\mathrm{x}_{1}$ and terminating at point $\mathrm{x}_{2}$. Extension to a $\mathrm{Y}$ axis Controller follows directly.

Since the $2500 \mathrm{~Hz}$ sampling rate is inadequate for determining precise switching points, an open-loop control strategy is adopted to alleviate the dependence of the control on the sampling rate. Of course, the inherent uncertainties of open-loop control dictate that a region of closed-loop control must be maintained to correct any maneuver errors that can arise as a result of inexact system modelling, external disturbances, or inaccuracies associated with the network. Thus, a hybrid open/closed-loop strategy referred to as the NN controller is proposed for conducting rest-to-rest maneuvers. Open-loop switching points are used to govern the nominal maneuver based on a $100 \mathrm{kHz}$ clock. After completion of the open-loop phase of the control, the original PD feedback control is used to remove any residual errors in position and velocity.

\subsection{Training Set Development}

This section presents a method for determining the switch times for maneuvering the fine stage in a time-optimal fashion. Since it is not practical to consider all possible maneuvers in order to generate a table of switch times as a function of initial and final conditions, the goal is to produce a set of optimal maneuvers that can be used to train an artificial neural network. Then, near optimal switch points can be obtained for an arbitrary maneuver by querying the neural network. Although it is applied only to the simulation in this study, this strategy does not depend on knowing an exact model of the system. Instead, the iterative scheme that determines the exact solutions used to train the network can be directly applied to the actual system. Thus, this approach has the added benefit of bypassing the modelling inaccuracies that plague open-loop control design.

\subsubsection{Time-Optimal Maneuver Strategy}

Because of the complex actuator dynamics, the open-loop time-optimal control requires two switch times $T_{s 1}$ and $T_{s 2}$. The first switch time signifies the beginning of the transition between saturated positive and saturated negative control. The second switch time allows for the finite time required for actuator shutdown. For maneuvers along the positive $\mathrm{X}$ axis beginning at $\mathrm{x}_{1}$, the time-optimal controller commands a full positive $\mathrm{X}$ modal control force for $0<t<T_{s 1}$, a full negative force for $T_{s 1}<t<T_{s 2}$, and a zero modal control force for $T_{s 2}<t<T_{f}$. Note that the final time $T_{s 3}$ is not an integral part of the openloop control strategy and is in general unspecified. If the switching points are valid, the velocity and the control force vanish as the system arrives at the target point $x_{2}$. The important parameters for a time-optimal rest-to-rest maneuver are designated by the pattern 
$\left\{\mathrm{x}_{1}, \mathrm{x}_{2}, \mathrm{~T}_{\mathrm{s} 1}, \mathrm{~T}_{\mathrm{s} 2}\right\}$. The final time is also included in the training pattern so that the network can provide an estimate of the total time required to complete a given maneuver. This estimate can be useful in defining a transition criterion for the open and closed-loop controllers.

In order to ensure that the full modal control force is utilized, the commanded force should be greater than the maximum force available with a minimal actuator gap for the range of motions considered. However, this over-specification should be minimal since it indirectly affects the remaining modal forces. From equations 2 and 3 , the actuator forces are calculated based on the desired modal forces. Therefore, an excessive $\mathrm{X}$ modal force can cause the actuators to overcompensate in an effort to maintain zero net torque about the $\mathrm{Y}$ axis. The end result is an inadvertent excitation of the out of plane motion. However, judicious selection of the desired modal force can minimize this problem. Again considering equations 2 and 3 , it is evident that two actuators combine efforts to produce $F_{x}(t)$. Previous experimental work revealed that each actuator can produce approximately $15 \mathrm{lbs}(66.72 \mathrm{~N})$ of force when the platen is centered in the frame. To ensure full utilization of the control authority while minimizing the out of plane excitation, the peak force demanded is set to $35 \mathrm{lbs}(155.68 \mathrm{~N})$.

\subsubsection{Determination of Training Points}

The training set should sufficiently cover the space of interest from -100 to +100 $\mu \mathrm{m}$ and should also include points beyond the range of interest. But since the endpoints of a training maneuver are arbitrarily chosen, the approach adopted in this study is to specify the initial position and the first switch time. Then, an iterative triangulation routine is used to determine the second switch time that produces a near zero velocity as the control force diminishes at the end of the control sequence. When this condition is satisfied, the final position is noted and the training pattern is complete. This procedure is summarized for translation in the positive $\mathrm{X}$ direction as follows:

1) Designate starting position $x_{1}$ and primary switch time $T_{s 1}$.

2) Initialize two switch times $T_{s 2 a}$ and $T_{s 2 b}$ that designate an interval containing the solution $T_{\mathrm{s} 2}$. The final velocities associated with the initial choices should satisfy $\mathrm{V}_{\mathrm{fa}}=\mathrm{V}_{\mathrm{f}}\left(\mathrm{T}_{\mathrm{s} 2 \mathrm{a}}\right)>0$ and $\mathrm{V}_{\mathrm{fb}}=\mathrm{V}_{\mathrm{f}}\left(\mathrm{T}_{\mathrm{s} 2 \mathrm{~b}}\right)<0$.

3) Set $T_{\text {s2old }}=T_{\text {s2new. }}$.

4) Let $T_{s 2 n e w}=\left(V_{f a} T_{s 2 b}-V_{f b} T_{s 2 a}\right) /\left(V_{f a}-V_{f b}\right)$. Round to nearest count of open-loop controller (see Figure 3).

5) If $\mid T_{\text {s2new }}-T_{\text {s2old }}=1 c c$ and $V_{f}\left(T_{\text {s2new }}\right) V_{f}\left(T_{\text {s2old }}\right)<0$, STOP.

6) Run simulation:

i) Command full positive modal force for $0<\mathrm{t}<\mathrm{T}_{\mathrm{sl}}$.

ii) Command full negative modal force for $T_{s 1}<t<T_{s 2 n e w}$.

iii) Command zero modal force for $T_{\text {s2new }}<$. 


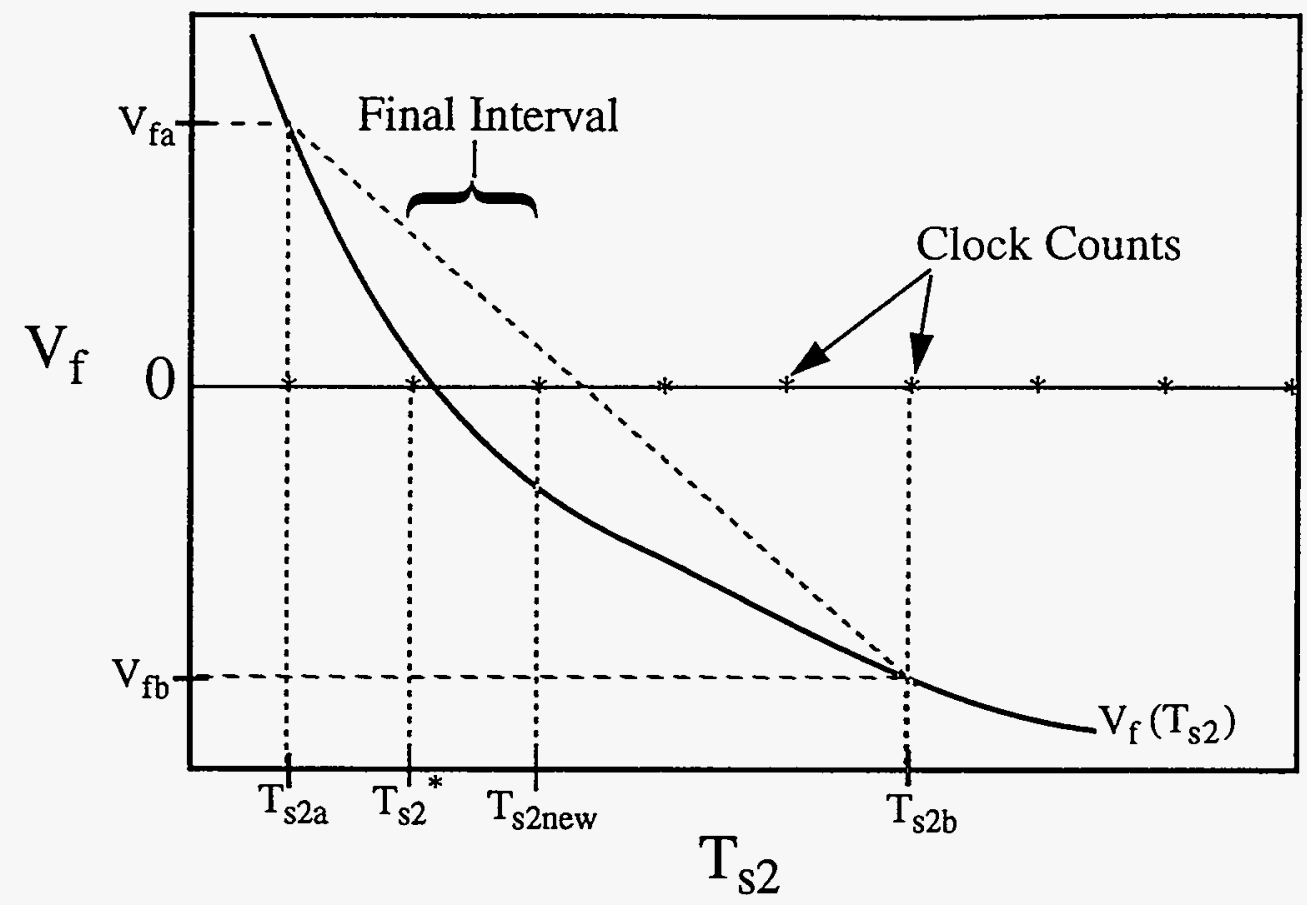

Figure 3. - Triangulation to Determine Second Switching Time.

iv) When the modal force is sufficiently small, note the final velocity $V_{f}$ and the final position $\mathrm{x}_{2}$.

7) If $\mathrm{V}_{\mathrm{f}}>0, \mathrm{~T}_{\mathrm{s} 2 \mathrm{a}}=\mathrm{T}_{\mathrm{s} 2 \mathrm{new}}, \mathrm{GO} \mathrm{TO} 3$.

8) If $\mathrm{V}_{\mathrm{f}}<0, \mathrm{~T}_{\mathrm{s} 2 \mathrm{~b}}=\mathrm{T}_{\mathrm{s} 2 \mathrm{new}}, \mathrm{GO} \mathrm{TO} 3$.

Convergence is obtained when the second switch time is narrowed to a one clock count interval for which the final velocity changes sign when using the switch times designated by the interval boundaries. The final switch time, denoted $\mathrm{T}_{\mathrm{s} 2}{ }^{*}$ in Figure 3 , is then taken as the interval boundary that produces the minimum magnitude final velocity. The associated final position $\mathrm{x}_{2}$ and final time $\mathrm{T}_{3 \mathrm{~s}}$ complete the training pattern. Prior to network training, one-half clock count adjustments are made to $\mathrm{T}_{\mathrm{s} 1}$ and $\mathrm{T}_{\mathrm{s} 2}$ so that the switch points fall within two distinct clock counts.

\subsubsection{Sample Training Point Determination}

Assuming a controller clock speed of $100 \mathrm{kHz}$ ( 1 clock count $(\mathrm{cc})=0.01 \mathrm{~ms})$, the triangulation routine is demonstrated in Figure 4 for a positive translation. With an initial position of $-10 \mu \mathrm{m}$, the primary switch point is set at $100 \mathrm{cc}(1.0 \mathrm{~ms})$ and the algorithm iterates on the second switch time in an effort to minimize the final velocity achieved when the control force drops below $0.00001 \mathrm{~N}$. After three iterations, the algorithm converges on a secondary switch time of $197 \mathrm{cc}(1.97 \mathrm{~ms})$. With this switching sequence, the table reaches a peak velocity of approximately $9641 \mu \mathrm{m} / \mathrm{sec}$ and comes nearly to rest with a residual velocity of approximately $27 \mu \mathrm{m} / \mathrm{sec}$. The convergence to the optimal modal force profile is illustrated in Figure 5. Actuator saturation in both the plus and minus direction is 


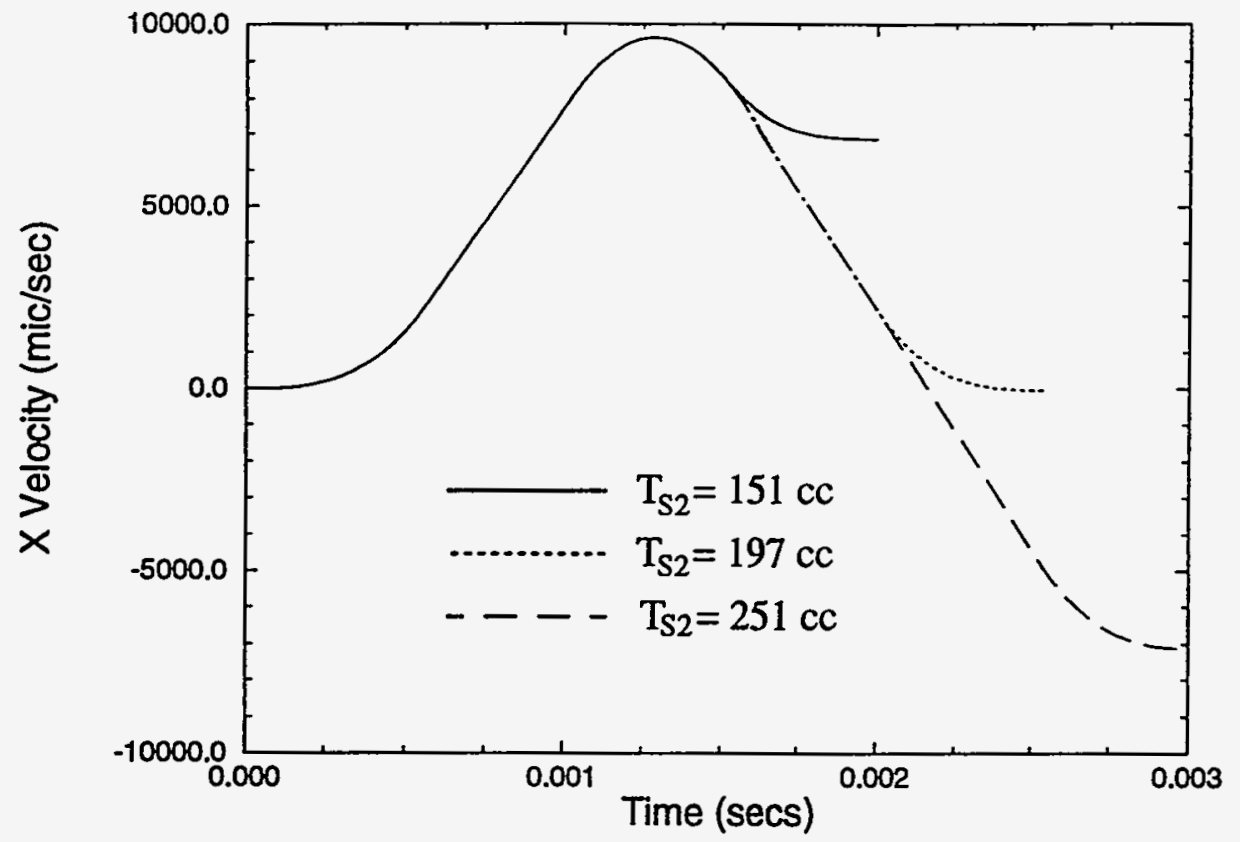

Figure 4. - Convergence to Optimal Velocity Profile.

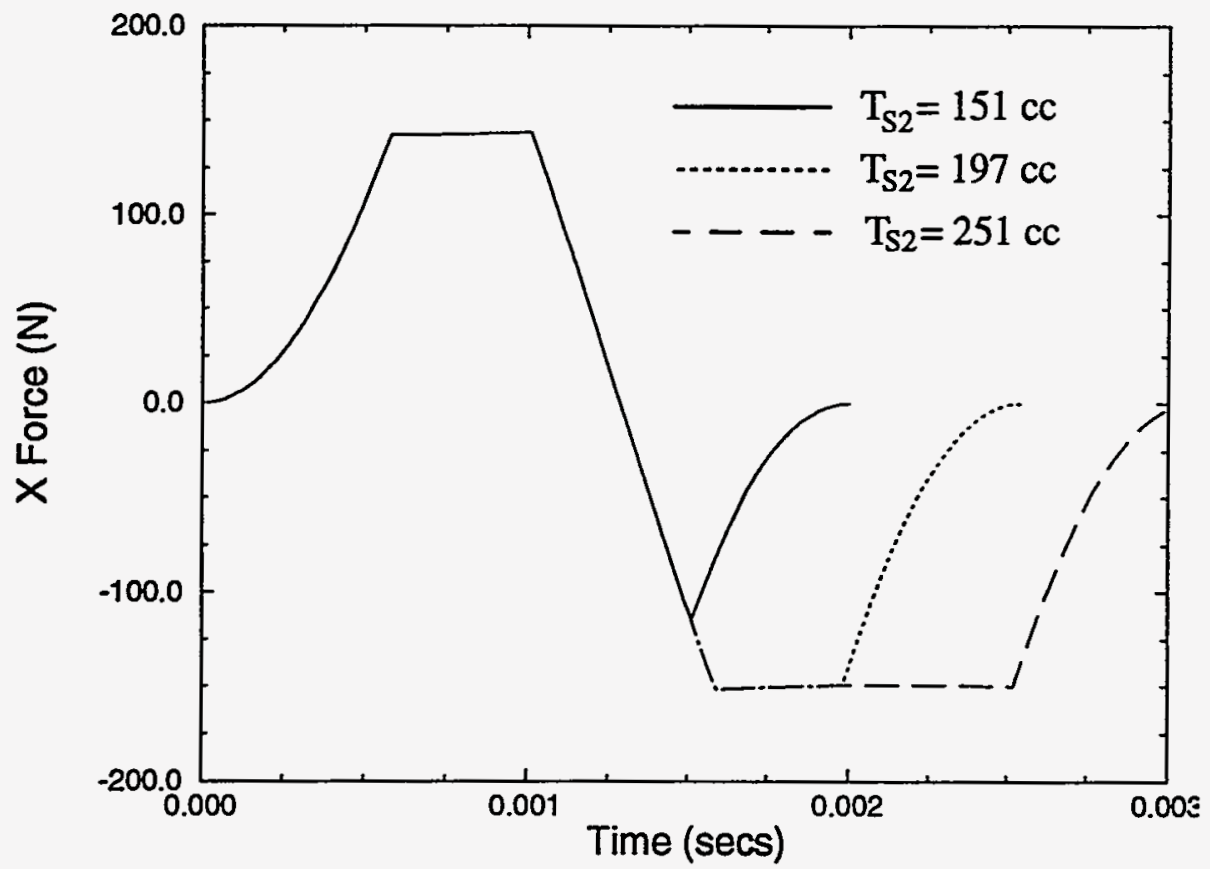

Figure 5. - Convergence to Optimal Force Profile.

evident, but the variation in saturation level as a function of position is barely perceptible since the resulting small maneuver is relatively small. The convergence to the optimal displacement profile is illustrated in Figure 6 . This example provides a single training pattern for the artificial neural network representing a maneuver from $-10 \mu \mathrm{m}$ to +0.069 $\mu \mathrm{m}$. A complete training set is obtained by repeating the procedure for a variety of initial positions and primary switch times. Several example training patterns are given in Table 2. Although it is not used in the open-loop controller, the final time can be used as a criterion 
for transition to the closed-loop disturbance rejection controller. For this reason, $T_{\mathrm{s} 3}$ is included in the training patterns. Also included in Table 2, the final velocities fall in the range of $+/-50 \mu \mathrm{m} / \mathrm{sec}$. Although these values are not included in the training patterns since they serve no useful purpose for the open-loop control, the final velocities provide an indication of the switching resolution. A faster clock could provide increased resolution and lower residual velocities, but these improvements would realistically be negated by increased noise in the controller circuit.

Table 2. - Sample Neural Network Training Patterns Using a 100kHz Clock.

\begin{tabular}{|c|c|c|c|c|c|}
\hline $\mathbf{X}_{\mathbf{1}}(\mu \mathbf{m})$ & $\mathbf{T}_{\mathbf{s 1}}(\mathbf{c c})$ & $\mathbf{T}_{\mathbf{s} 2}(\mathbf{c c})$ & $\mathbf{T}_{\mathbf{s} 3}(\mathbf{c c})$ & $\mathbf{X}_{\mathbf{2}}(\mu \mathbf{m})$ & $\mathbf{V}_{\mathbf{f}}(\mu \mathbf{m} / \mathbf{s})$ \\
\hline \hline-110.00 & 500.5 & 911.5 & 968 & 69.15 & -20.0 \\
\hline-38.51 & 207.5 & 381.5 & 438 & 0.81 & -47.9 \\
\hline-28.73 & 126.5 & 229.5 & 286 & -14.62 & 45.8 \\
\hline 72.63 & 89.5 & 233.5 & 290 & 85.66 & 4.8 \\
\hline 53.74 & 92.5 & 228.5 & 285 & 66.59 & -10.69 \\
\hline-100.00 & 502.5 & 1013.5 & 1070 & 109.71 & -18.5 \\
\hline-112.67 & 44.5 & 72.5 & 101 & -112.29 & 22.4 \\
\hline 4.76 & 222.5 & 556.5 & 613 & 83.24 & 7.6 \\
\hline
\end{tabular}



Figure 6. - Convergence to Optimal Displacement Profile. 


\subsection{Neural Network Design}

A network was required that takes as inputs the desired beginning and endpoints of a translation and outputs the appropriate switching times for the controller. By design, the resolution of the training and testing data for the neural network is one count of the 100 $\mathrm{KHz}$ switching clock. The goal in training the network is to get as close as possible to the ideal error of $+/-0.5$ clock counts. Since the range of outputs for $T_{s 1}, T_{s 2}$, and $T_{s 3}$, respectively, were 27.5 to $550.5,44.5$ to 1182.5 , and 62.5 to 1239 clock counts, this posed a challenging problem for a backpropagation network. Thus a General Regression Neural Network (GRNN) was chosen for the job (Ref. 15).

\subsubsection{General Regression Neural Networks}

General Regression Neural Networks (GRNN's) utilize radial basis functions, nodes whose range of influence is limited to a small region of the input space, as their nonlinear element. Advantages of the GRNN approach are that minimal training is required, prototype nodes may be added without great disturbance to the existing knowledge of the network, and that arbitrary accuracy is more easily obtained from GRNN's than from backpropagation networks. The primary disadvantages of this type of network are that they require much more storage space and computation time than a similarly trained backpropagation network.

The GRNN equations are as follows

$$
\hat{y}(\bar{x})=\frac{\sum_{p} \overline{y_{p}} w\left(\bar{x}, \overline{x_{p}}\right)}{\sum_{p} w\left(\bar{x}, \overline{x_{p}}\right)}
$$

where $\bar{x}$ is the current network input, $p$ is the prototype index, $\bar{x}_{p}$ is the prototype input vector, $\overline{y_{p}}$ is the prototype output vector, $\hat{y}(\bar{x})$ is the estimate of $y$ for input vector $\bar{x}$, and $w$ is the radial basis function or window function for the network. For this work, a decaying exponential was used as a basis function

$$
w\left(\bar{x}, \bar{x}_{p}\right)=\exp \left(\frac{\bar{x}^{T} \bar{x}_{p}}{2 \sigma^{2}}\right)
$$

in which $\sigma$ is a constant specifying the range of influence of each prototype node.

\subsubsection{GRNN Optimization}

The accuracy requirements for this network caused the size of the training set to be very large. With the GRNN technique, in general, the entire training set is taken to be the prototype set of the network. In an effort to reduce the size of the network, a "trimming" algorithm was written that eliminates those prototypes that do not improve the accuracy of 
the network. An unexpected benefit of this trimming algorithm occurred: the accuracy of the network actually improved as selected points were eliminated from the training set. The algorithm proceeds as follows:

Inputs: $\mathrm{XP}$ and YP are training inputs and outputs, itrn indicates indices of initial prototype set. ERRTOL - acceptable error bound. PASSES - Number of times to pass through data.

1. Calculate NUM_NANS and SUM_EXCESS_ERROR,

NUM_NANS - number of Not A Number results obtained in executing network on entire training set. (These occur as a result of divide by zero errors when there are no prototype points near an input point).

SUM_EXCESS_ERROR - sum of absolute values of errors in excess of ERRTOL

2. For each PASS

A. Create an experimental prototype set by adding a randomly selected member of the training set to or subtracting it from the existing prototype set.

B. Evaluate effect on NUM_NANS and SUM_EXCESS_ERROR

C. Decide whether to keep experimental prototype set or revert to previous. If NUM_NANS or SUM_EXCESS_ERROR improved, keep the experimental set.

D. GOTO A until every vector in the training set has been evaluated.

3. GOTO 2 until specified number of passes is completed or the current pass resulted in no changes to the prototype set.

\subsubsection{Network Specifics}

Independent training and test sets were assembled consisting of 12546 and 309 moves, respectively. The starting and ending locations were preprocessed as shown in Figure 7 so that the GRNN network had three inputs and three outputs. The outputs were T1 (switch time \#1 in clock counts), T2 (switch time \#2 in clock counts), and T3 (completion time for open loop move). The trimming algorithm reduced the training set to 9154 prototype nodes. This configuration of the network required 302,084 flops to process one set of inputs. An alternate implementation which disregards very distant prototype points required approximately $28 \%$ of the computation while producing the same results. 


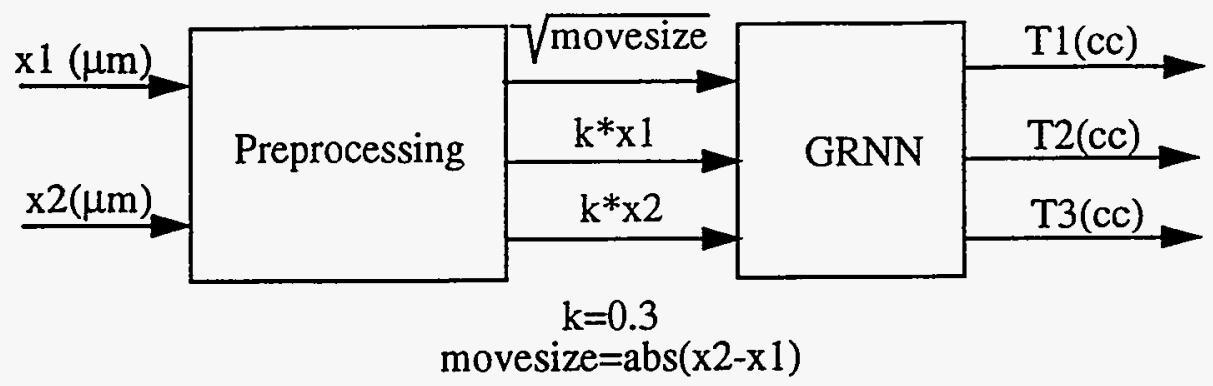

Figure 7. - Switch Time Estimation Flow Diagram.

\subsubsection{Network Error Summary}

While the goal of the training was to provide good estimates over the range of movesizes between 1 and 180 microns, data outside this region is necessary to achieve good performance over the entire target region. Table 3 shows how many training and test samples fell in each range of movesizes. Figures 8 through 10 show the maximum and minimum errors that occurred in each of these ranges. It is notable that in general the maximum errors on the test set are smaller than those obtained for the training set. For a test set of sufficient size, the errors would be nearly identical, indicating excellent generalization capability of the network. The exception to this occurs for the small maneuver sizes, where the minimum error becomes large. This is due to the tendency in this region for there to be more larger moves affecting the computation of the switch points than smaller moves. However, in the region of interest, $(>=1 \mu \mathrm{m})$, the peak errors plateau between 2 and 3 clock counts.

Table 3. - Network Training Data Summary.

\begin{tabular}{|c|c|c|c|}
\hline $\begin{array}{c}\text { range_lo } \\
(\mu \mathrm{m})\end{array}$ & $\begin{array}{c}\text { range_hi } \\
(\mu \mathrm{m})\end{array}$ & $\begin{array}{c}\text { \# train } \\
\text { points }\end{array}$ & $\begin{array}{c}\text { \# test } \\
\text { points }\end{array}$ \\
\hline \hline 0.01 & 0.25 & 225 & 11 \\
\hline 0.25 & 0.50 & 267 & 17 \\
\hline 0.50 & 1.00 & 346 & 42 \\
\hline 1.00 & 2.00 & 414 & 22 \\
\hline 2.00 & 5.00 & 1040 & 17 \\
\hline 5.00 & 10.00 & 1253 & 22 \\
\hline 10.00 & 20.00 & 1666 & 15 \\
\hline 20.00 & 40.00 & 2143 & 34 \\
\hline 40.00 & 80.00 & 2481 & 57 \\
\hline 80.00 & 100.00 & 790 & 22 \\
\hline
\end{tabular}




\begin{tabular}{|c|c|c|c|}
\hline $\begin{array}{c}\text { range_lo } \\
(\mu \mathrm{m})\end{array}$ & $\begin{array}{c}\text { range_hi } \\
(\mu \mathbf{m})\end{array}$ & $\begin{array}{c}\text { \# train } \\
\text { points }\end{array}$ & $\begin{array}{c}\text { \# test } \\
\text { points }\end{array}$ \\
\hline \hline 100.00 & 120.00 & 599 & 20 \\
\hline 120.00 & 140.00 & 419 & 12 \\
\hline 140.00 & 160.00 & 306 & 10 \\
\hline 160.00 & 180.00 & 224 & 7 \\
\hline 180.00 & 200.00 & 171 & 1 \\
\hline 200.00 & 220.00 & 101 & 0 \\
\hline 220.00 & 240.00 & 68 & 0 \\
\hline
\end{tabular}

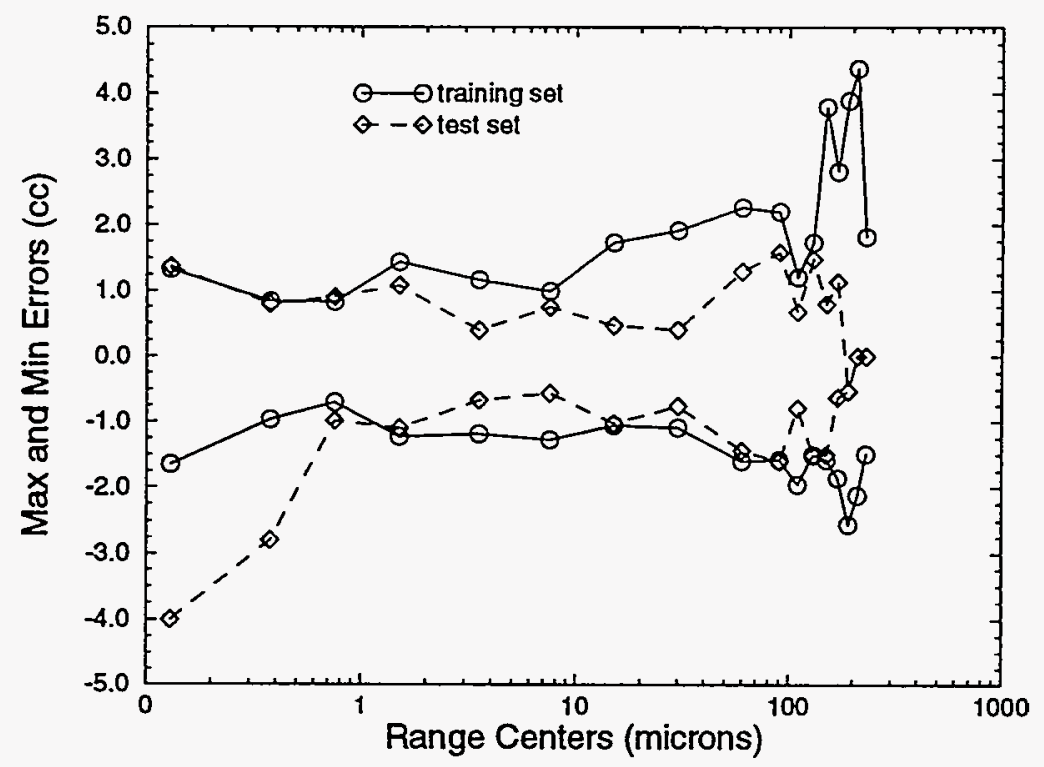

Figure 8. - $T_{s 1}$ Error Bounds as a Function of Movesize. 




Figure 9. - $T_{\mathrm{s} 2}$ Error Bounds as a Function of Movesize.

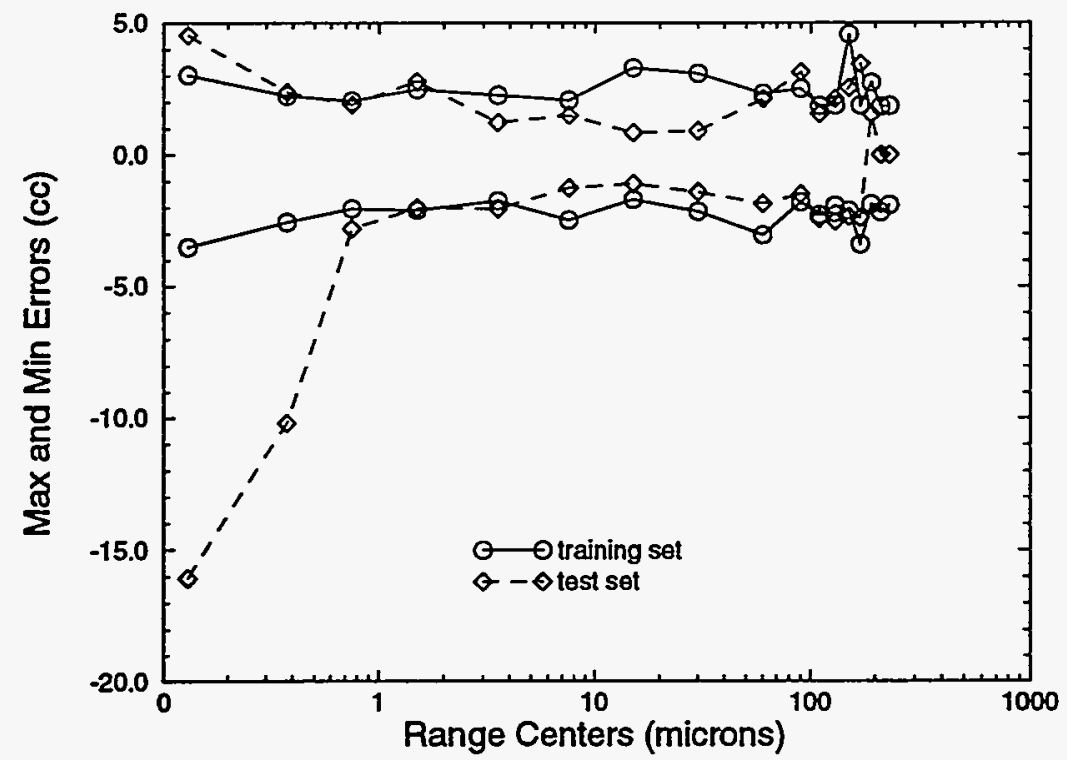

Figure 10. - $T_{s 3}$ Error Bounds as a Function of Movesize. 


\subsection{CONTROLLER DEMONSTRATIONS}

In this section, the performance of each controller is assessed through repeated simulations. As in the development sections, the system is considered free of noise, and no external disturbances interfere with the maneuvers. One dimensional maneuvers along the $X$ axis are first considered to illustrate each controller's ability to complete rest-to-rest maneuvers over a broad range of motion. Results highlight the NN controller's superiority in terms of settling time. A simultaneous X-Y maneuver is then considered to illustrate each controller's ability to integrate the two distinct step commands. Although the NN controller again proves to be the most efficient in terms of settling time, it has the undesirable consequence of increased excitation of the out of plane motion.

\subsection{0ne Dimensional X Translations}

To illustrate the effectiveness of the three control strategies, we first considered one dimensional maneuvers along the $\mathrm{X}$ axis. As explained in section 3, the bandwidth of the original PD controller is limited to $50 \mathrm{~Hz}$ to prevent actuator saturation over a wide range of possible maneuvers. Since this is not the limiting concern in the TOC controller, the bandwidth used to define the boundaries of region $R$ is increased to $90 \mathrm{~Hz}$. A peak modal force of $30 \mathrm{lbs}$ was assumed since two actuator pairs drive the motion along the $\mathrm{X}$ axis, and a conservative scaling factor of $\alpha=0.8$ was used to minimize the deviations from the desired phase trajectory to the origin. After repeated simulations, the transition time used to define the onset switch curve was set to $1.5 \mathrm{~ms}$ to accommodate actuator dynamics and the relatively coarse sampling period. This coarse sampling period leads to the implementation of an open-loop strategy for the NN controller as described in the previous section. For the $\mathrm{NN}$ controller, transition to the original $50 \mathrm{~Hz}$ PD controller occurred after the second switch time when the magnitude of the velocity dipped below $300 \mu \mathrm{m} / \mathrm{sec}$. In the event that the network error was too substantial to permit satisfaction of this criterion, the PD control was activated when the elapsed time exceeded the final time predicted by the NN controller.

\section{$5.1 .1150 \mu \mathrm{m}$ Maneuver}

The displacement profiles generated by the three controllers for a $150 \mu \mathrm{m}$ maneuver beginning at $-75 \mu \mathrm{m}$ and terminating at $75 \mu \mathrm{m}$ are shown in Figure 11. As compared to the PD controller, the TOC and NN controllers provided 50\% and 75\% reductions in the settling time, respectively. The enhanced performance was the direct result of increased utilization of the available control force as illustrated in Figure 12. While the PD control input was typical of a critically damped system, the TOC control input revealed some interesting features. A rapid increase in the control force was followed by a period of saturation, with the saturation level increasing as the platen moved along the $\mathrm{X}$ axis toward the positive actuators. A rapid switch to the conservative force estimate was initiated at approximately $4 \mathrm{~ms}$ as the trajectory entered region $S$ in the phase plane. A slight variation in the peak force supplied by the negative actuators was revealed as the actuator gaps lengthened between sampling points. As a consequence of the coarse sampling rate, the phase trajectory initially missed region $R$ and exited region $S$, essentially resetting the controller. A second region of positive control saturation was quickly followed by a second region of conservative negative control before the trajectory entered region $R$ and the PD 




Figure 11. - X Modal Displacement History for $150 \mu \mathrm{m}$ Maneuver.

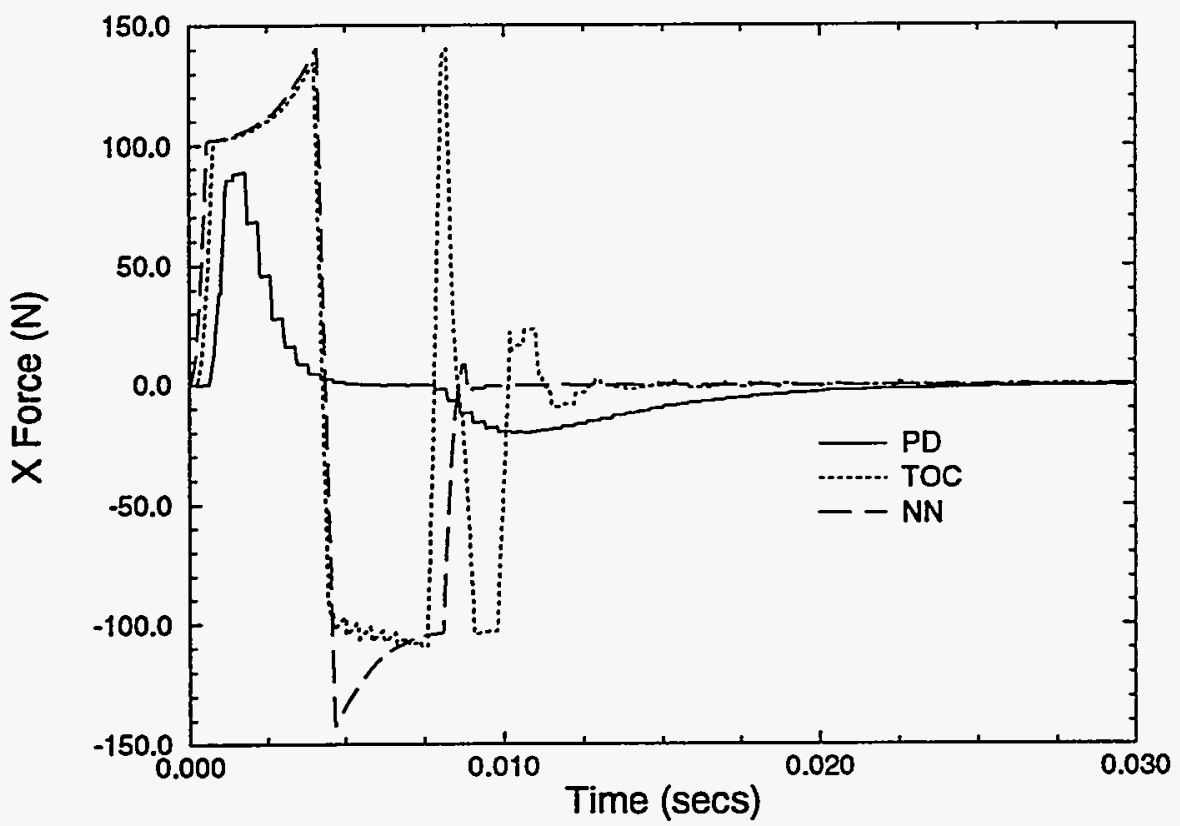

Figure 12. - X Modal Force History for $150 \mu \mathrm{m}$ Maneuver.

control was activated. The rapid switching of the control caused inflections in the displacement profile near $8 \mathrm{~ms}$. In comparison, the NN controller was much more efficient than the TOC controller since the open-loop strategy enabled precise designation of the switch points and eliminated the need for conservative force estimates. With $\mathrm{T}_{\mathrm{s} 1}$ equal to $4.09 \mathrm{~ms}$, a positive saturation period was followed by a negative saturation in which the saturation level decreased as the platen moved away from the negative actuators. The 


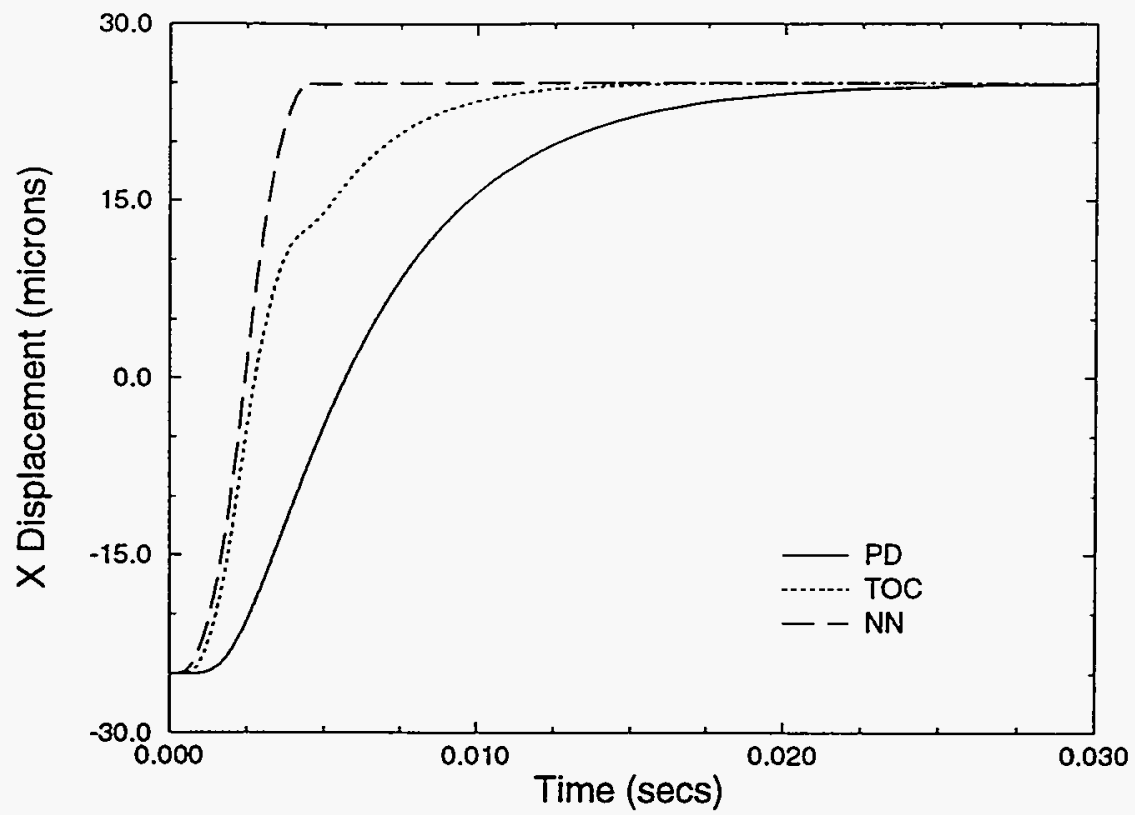

Figure 13. - X Modal Displacement History for $\mathbf{5 0} \mu \mathrm{m}$ Maneuver.

control was shut off after $\mathrm{T}_{\mathrm{s} 2}$ equal to $8.12 \mathrm{~ms}$, and the platen coasted toward the target point with only a minimal $P D$ correction required.

\section{$5.1,250$ um Maneuver}

The displacement profiles for a maneuver beginning at $-25 \mu \mathrm{m}$ and terminating at $+25 \mu \mathrm{m}$ are given in Figure 13. Reductions in settling time of approximately $50 \%$ and $85 \%$ were achieved using the TOC and NN controllers, respectively. Although the profile was smoother than in the previously considered maneuver, an inflection in the displacement was again evident for the TOC controller near $5 \mathrm{~ms}$. In this case, the coarse sampling of the phase trajectory caused a deviation that from the desired path and the trajectory entered region $R$ near the upper left corner. A mismatch of the desired control force on either side of the boundary to $R$ at this location caused an abrupt change in the control force as shown in Figure 14. Conversely, the precise switching provided by the NN controller at 2.16 and $4.31 \mathrm{~ms}$ produced a smooth transition from the initial to the terminal points.

\section{$5.1 .32 \mu \mathrm{m}$ Maneuver}

Finally, we considered a small maneuver, beginning at $-1 \mu \mathrm{m}$ and terminating at +1 $\mu \mathrm{m}$. The displacement profiles for the three controlled cases are shown in Figure 15 and the corresponding modal control forces are given in Figure 16. Although the resolution of the simulation makes it difficult to pinpoint an exact settling time in each case, a significant reduction was realized using the NN controller with switching times of 0.53 and $1.06 \mathrm{~ms}$. Some reduction in settling time was also evident for the TOC controller, but this is an artificial improvement. Due to the magnitude of the maneuver, the initial position in the phase plane was contained in $R$. As a consequence, the PD control was immediately activated and governed the entire maneuver. Thus, the performance achieved using the 


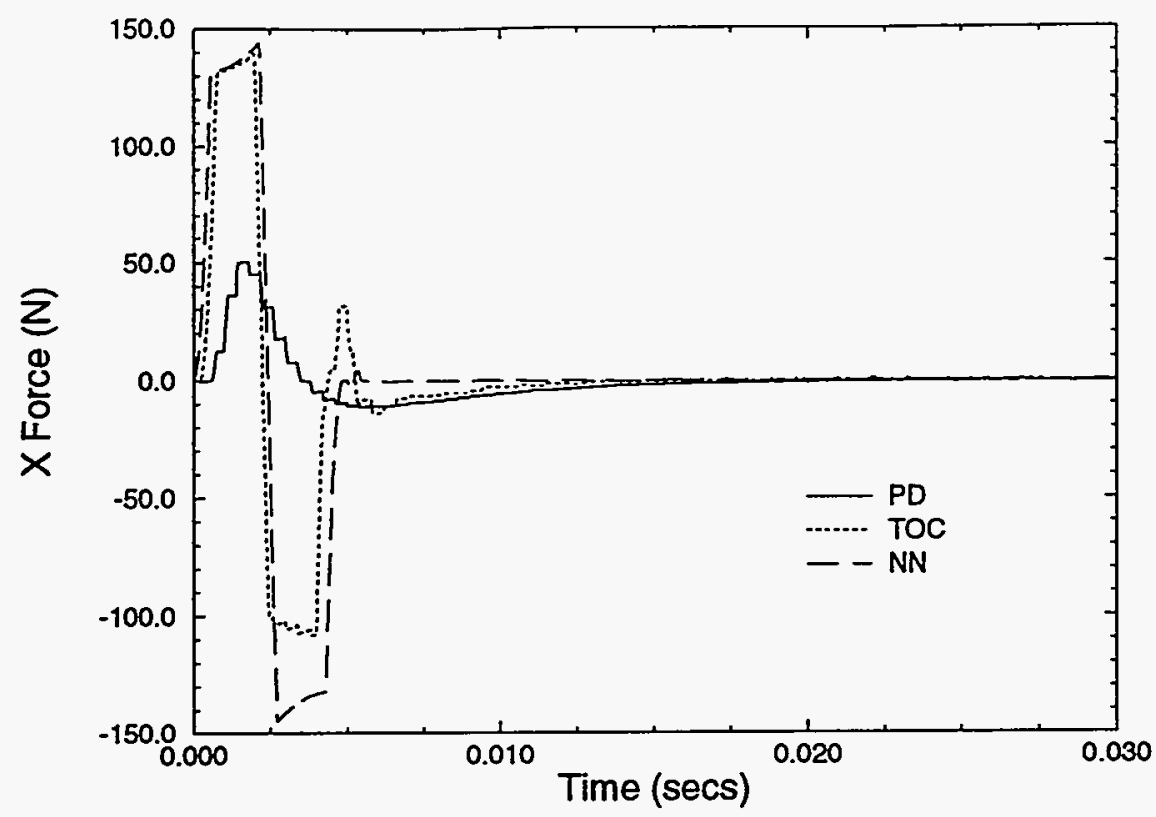

Figure 14. - X Modal Force History for $50 \mu \mathrm{m}$ Maneuver.

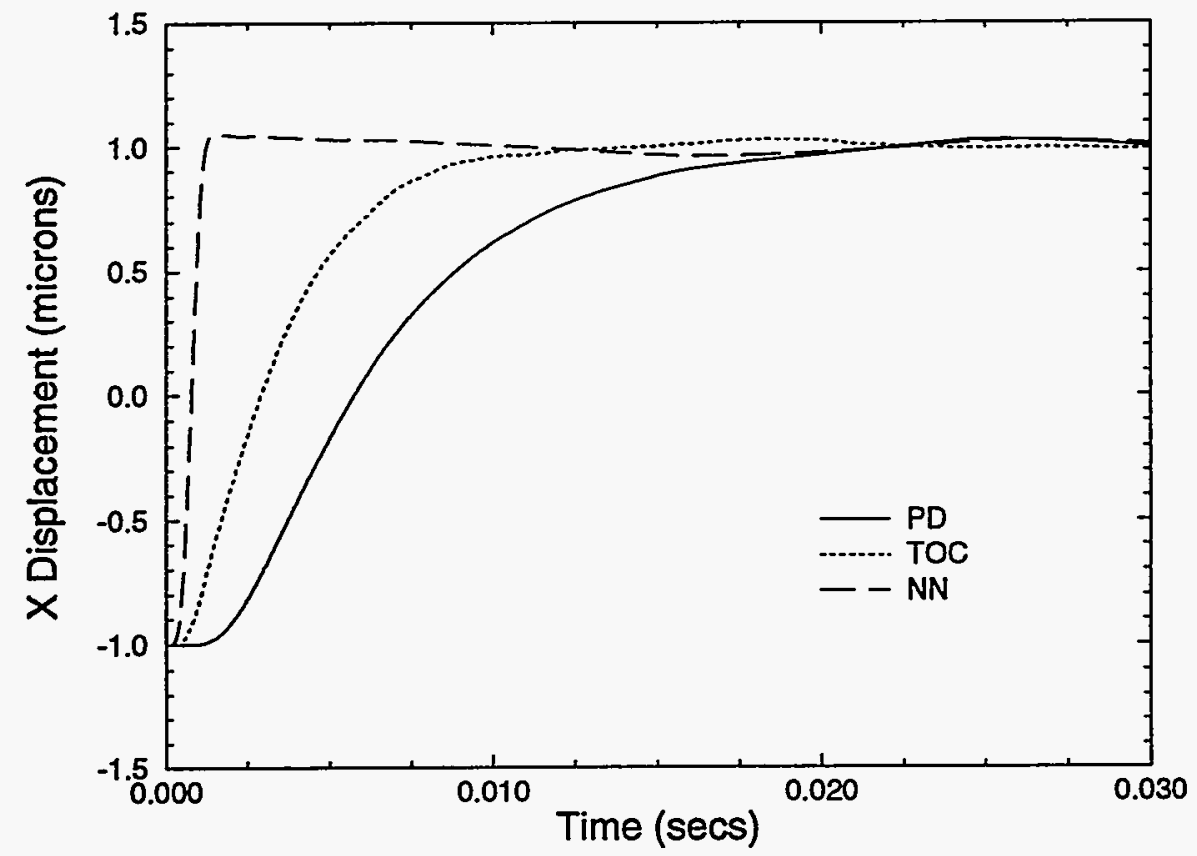

Figure 15. - X Modal Displacement History for $2 \mu \mathrm{m}$ Maneuver.

TOC controller could be matched by the original controller by simply increasing its bandwidth. Although this may result in actuator saturation for large maneuvers, this is an acceptable solution to improving performance for small maneuvers near the origin.

\subsubsection{Average Performance}

The relative performances of the three controllers are best evaluated by considering the average settling times produced for a variety of maneuvers throughout the range of possible motion. Thus the simulation was repeatedly run using each controller with 


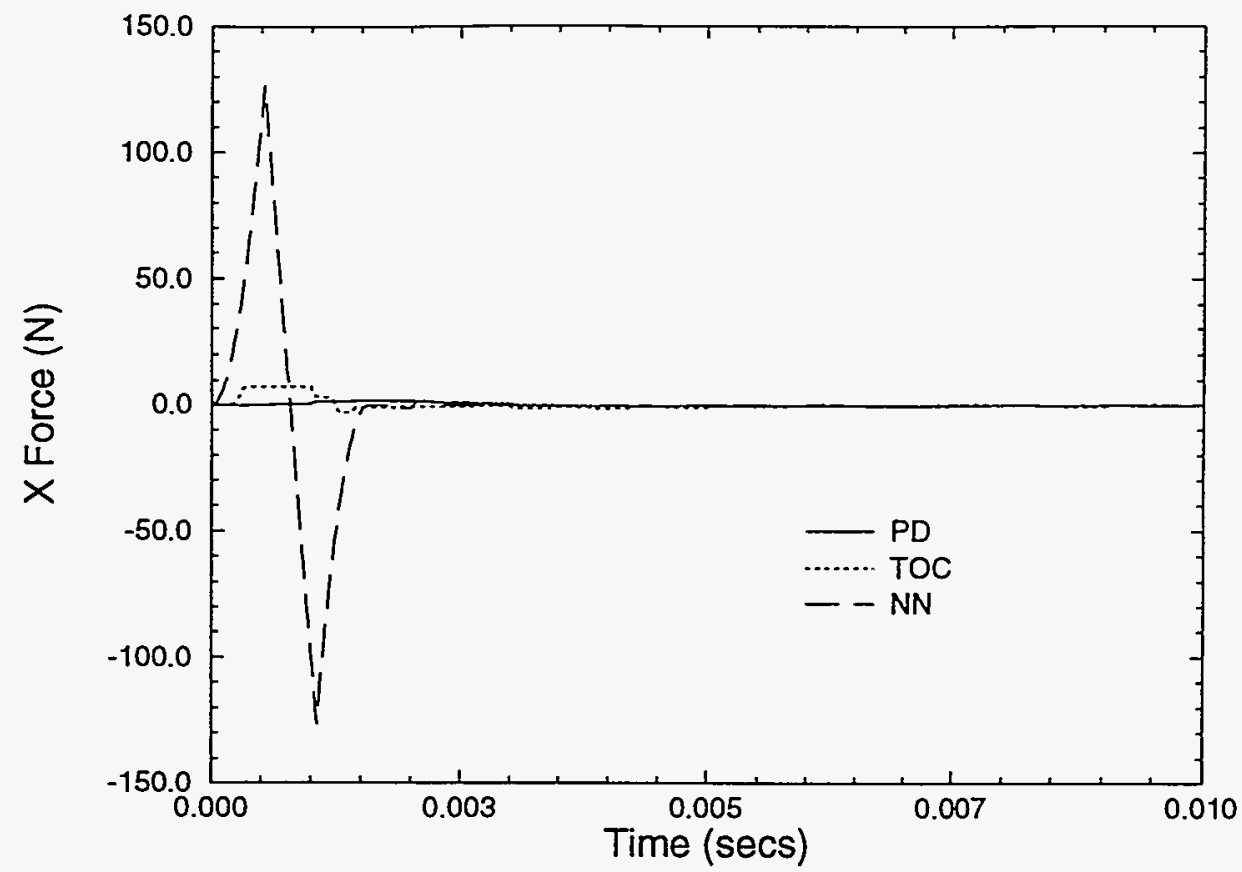

Figure 16. - X Modal Force History for $2 \mu \mathrm{m}$ Maneuver.

randomly generated starting positions in the range of $+/-100 \mu \mathrm{m}$. The final positions were determined by randomly generated movesizes in both the positive and negative directions. The move sizes were incremented in steps of $10 \mu \mathrm{m}$, beginning with random maneuvers in the 0 to $+/-10 \mu \mathrm{m}$ range and ending with the $+/-140$ to $+/-150 \mu \mathrm{m}$ range. Thus, terminal positions of $+/-250 \mu \mathrm{m}$ were possible. Fifty runs were made for each $10 \mu \mathrm{m}$ increment in the move size for a total of 750 runs with each controller and the settling times were recorded. The settling time was defined as the elapsed time from the beginning of the maneuver until the $\mathrm{X}$ modal displacements settled at the target point within $1 \%$ of the movesize.

The average settling times as a function of the average movesize are shown in Figure 17. As expected, the average settling times for the PD controller are relatively constant throughout the range of motion. A slight increase in the settling time is evident for movesizes less than $10 \mu \mathrm{m}$ and greater than $80 \mu \mathrm{m}$. For small maneuvers, the resolution of the simulation inhibits the controller's ability to maintain the final position within $1 \%$ of the movesize in some cases. In these instances, a settling time of $30 \mathrm{~ms}$ was assumed, boosting the average settling time for small maneuvers. Actuator saturation occurred for many of the larger maneuvers causing a modest increase in the average settling times.

The TOC controller showed relatively good performance for move sizes between 0 to $100 \mu \mathrm{m}$. Again, the controller for small move size is equivalent to the PD control with increased bandwidth since the entire maneuver occurs in region $R$. An increase in the settling time for small maneuvers resulted from the limited resolution of the simulation. In cases where the settling time could not be clearly identified, it was set to $20 \mathrm{~ms}$. For average move sizes larger than $100 \mu \mathrm{m}$, some cases with extreme terminal positions exhibited severe actuator saturation, causing the phase trajectory to deviate drastically from the desired path. In some cases, region $R$ was missed completely and oscillatory behavior 


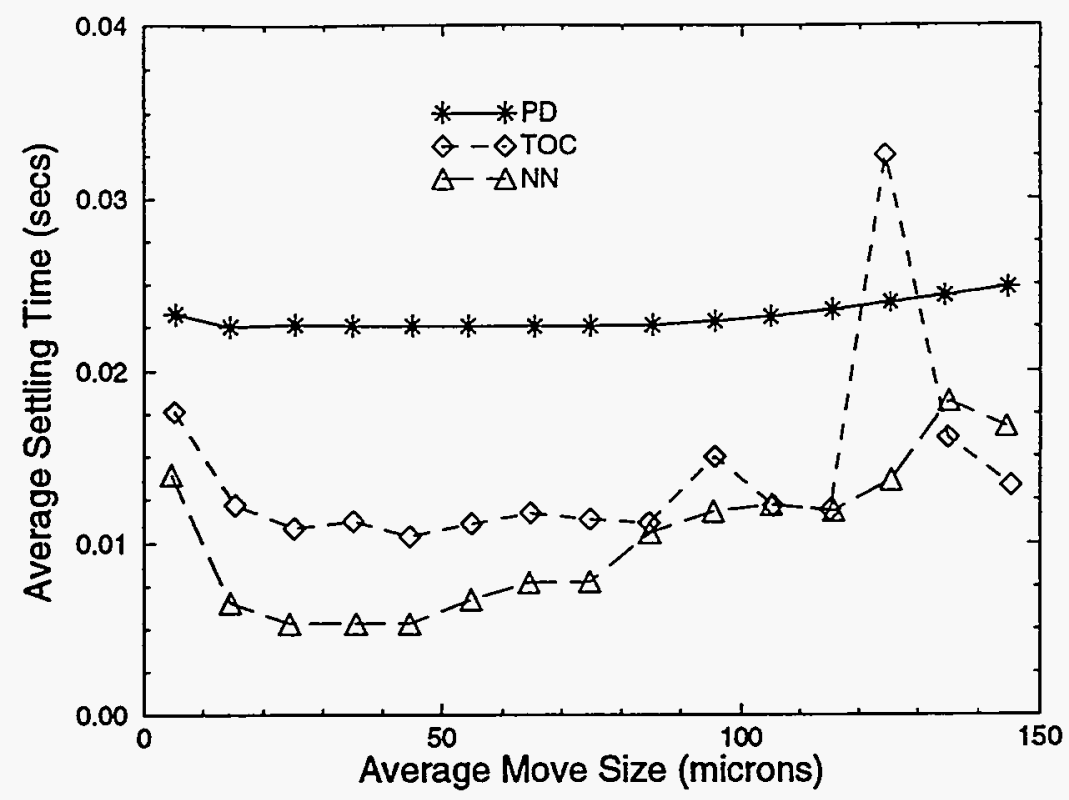

Figure 17. - Comparison of Controller Performances for

\section{One Dimensional Maneuvers.}

resulted. This occurred most frequently for move sizes between 120 and $130 \mu \mathrm{m}$ and accounts for the sharp increase in average settling time.

The NN controller displayed vastly superior performance up to approximately 130 $\mu \mathrm{m}$. For small maneuvers in which the settling time was not clearly delineated, $1.5 \mathrm{~ms}$ was assumed. Since the NN was trained using data in the region of $+/-100 \mu \mathrm{m}$, a degradation in the settling time was seen for large moves which were more likely to include regions outside the training set. However, for move sizes up to $60 \mu \mathrm{m}$, the TOC and NN controllers provided approximately $50 \%$ and $70 \%$ reductions in settling time as compared to the original PD controller.

\subsection{Combined X and Y Repositioning}

Since the role of the fine stage is generally to correct for coarse stage positioning errors in the plane while regulating out of plane motion, we next considered simultaneous $\mathrm{X}$ and $\mathrm{Y}$ maneuvers. Note that these two modes do not share common actuator pairs. Therefore the one dimensional control strategies can be applied to both the $\mathrm{X}$ and $\mathrm{Y}$ maneuvers simultaneously without increasing the likelihood of actuator saturation. Figure 18 shows the results of a move from $-53 \mu \mathrm{m}$ to $4 \mu \mathrm{m}$ along the $\mathrm{X}$ axis and from $-10 \mu \mathrm{m}$ to $32 \mu \mathrm{m}$ along the $\mathrm{Y}$ axis. Although the TOC displacement again encountered the inflections that are characteristic of multiple switching caused by missing region $R$, some improvement in settling time was realized as compared to the PD controller. The NN controller commanded switches at 2.61 and $4.66 \mathrm{~ms}$ for the $X$ motion and 1.28 and $2.85 \mathrm{~ms}$ for the $Y$ motion. The displacement profiles show significant improvements in the settling times for both the $\mathrm{X}$ and $\mathrm{Y}$ motion. 


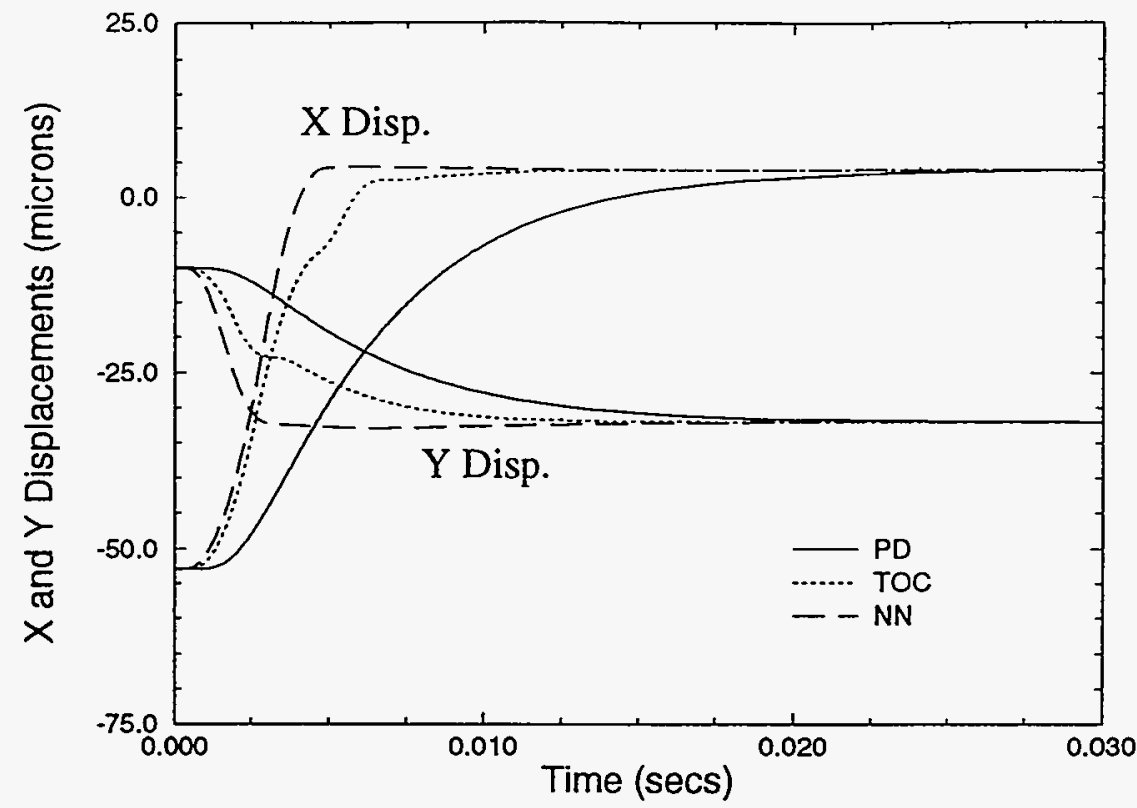

Figure 18. - Two Dimensional Maneuver Displacement Histories.

\subsection{Out of Plane Motion}

In the previously described examples, the $\mathrm{NN}$ and TOC controllers provided improved settling times over a wide range of translation maneuvers. However, the lack of feedback control prior to transition to PD control for the NN and TOC controllers resulted in uncompensated out of plane motion. As an example, the rotations about the $\mathrm{X}$ axis for the two dimensional translation case are shown in Figure 19. While the PD control caused very little excitation of this mode due to its feedback compensation on all axes, both the NN and TOC controllers produced significant levels of excitation. For the TOC controller, the excitation primarily resulted from the coupling of the modes through the actuators. Since the $Y$ actuators' line of action is not coincident with the center of mass, the platen rotates about the $\mathrm{X}$ axis during a $\mathrm{Y}$ step maneuver. This inadvertent excitation is partially compensated, however, since the actuators on the top and bottom sides of the platen provide a counter moment in an effort to maintain a zero net torque about the $\mathrm{X}$ axis. When the trajectory entered $R$, the PD control was activated for all modes and this undesirable motion was eliminated. For the NN controller, an even higher level of out of plane motion was realized with a peak rotation of $-23 \mu \mathrm{rads}$. In addition to the coupling phenomenon, this excitation resulted from the actuator saturation that forms the basis of the control strategy. Recall that the desired actuator pair forces are computed on the basis of the desired modal forces according to equation 2. To ensure full utilization of the available actuator forces, the desired $\mathrm{Y}$ modal force was over-specified during the maneuver. In response, the actuators on the top and bottom of the platen overcompensate while trying to maintain zero net torque about the $\mathrm{X}$ axis. The end result was additional excitation of the out of plane motion. The amount of overcompensation was minimized by limiting the excess modal force demanded yet still ensuring full actuator utilization. Compensation for out of plane motion was initiated at the termination of the open-loop phase of the control. 


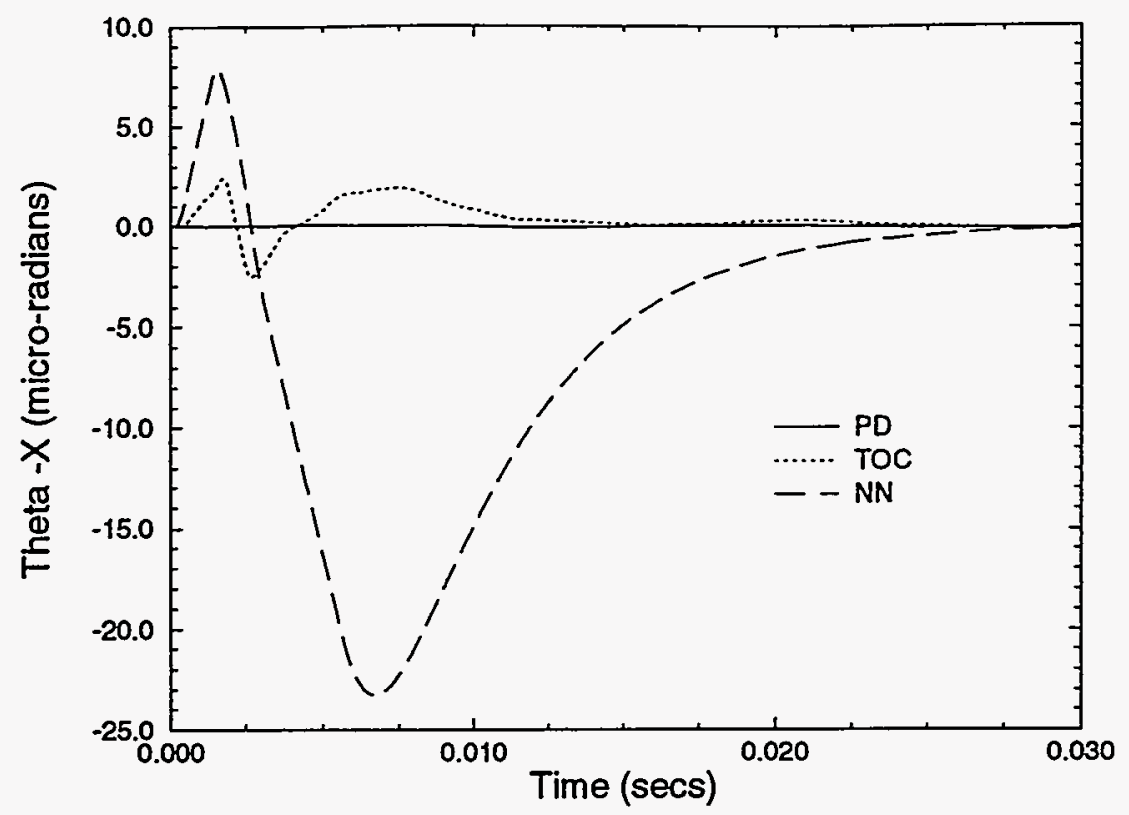

Figure 19. - X Axis Rotation for Two Dimensional Maneuver.

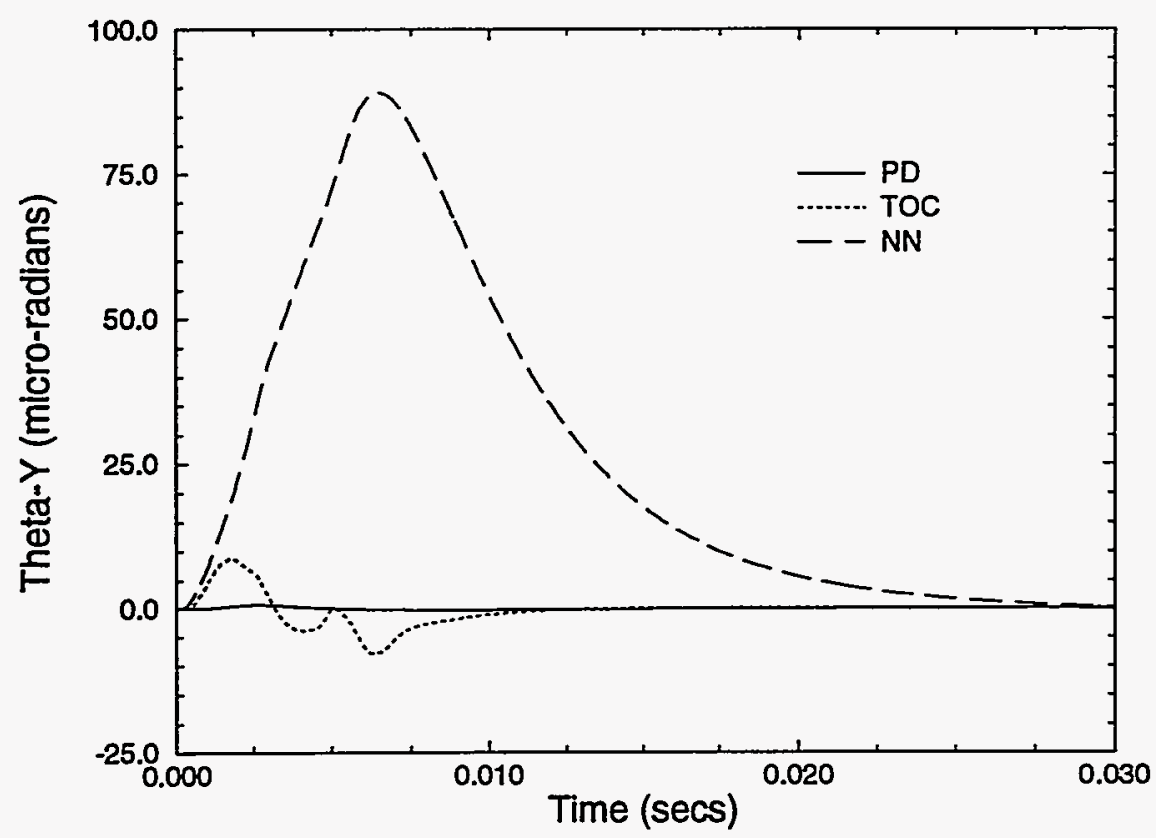

Figure 20. - Y Axis Rotation for Two Dimensional Maneuver.

Similar arguments hold for the rotation about the $\mathrm{Y}$ axis as shown in Figure 20. Only a negligible amount of excitation was evident using the PD control while the TOC and NN control showed increasing levels of excitation. This undesirable motion persisted until the activation of the PD control which compensated for motion in all six degrees of freedom. Although excessive out of plane motion can adversely affect the focusing of the photolithography system, the significance of the out of plane motion for typical maneuvers 
is yet unknown. If necessary, further modifications of the controllers are possible to provide some closed-loop compensation of the out of plane motion while conducting the $\mathrm{X}$ and $\mathrm{Y}$ open-loop translations. 


\subsection{RECOMMENDATIONS FOR FUTURE WORK}

Before the time-optimal controllers can be implemented on the magnetically levitated fine stage, their effects on the out of plane excitation and the vibrational characteristics of the platen must be further investigated. The least critical of the two issues is the out of plane excitation. Although large motions can cause focusing problems that impede the manufacture of ICs, this issue has little potential for harming the system. The effects of the rapid actuator switching on the flexible body motion, however, is a much more critical problem. The interaction of the controller with the platen structural dynamics was ignored in this study in order to focus on the issues related to time-optimal positioning of magnetically levitated systems. Nevertheless, the platen flexibility can cause instability, presenting the potential for harming the stage.

\subsection{Compensating for Out of Plane Motion}

As previously mentioned, both the TOC and NN controllers inadvertently generate undesirable motion of the platen while conducting typical X-Y translational maneuvers. Further study of this motion will gauge its significance in terms of the minimum feature size that can be reproduced on the IC. If this motion adversely affects the quality of chips, then the time-optimal control strategies should be augmented with closed-loop compensation for all six rigid body DOFs.

\subsection{Compensating for Platen Vibrations}

The focus of this study was to develop time-optimal control techniques applicable to a system possessing significant nonlinearities in the actuator dynamics. Therefore, platen flexibility was ignored in order to focus attention on the complexities of the actuation hardware. However, the real system has been shown to exhibit significant structural flexibility. Several lightly damped flexible body modes of a two-piece platen/mirror structure have been encountered during operation. These modes limit the rigid body controller bandwidth to approximately $30 \mathrm{~Hz}$, beyond which the excitation of the flexible modes causes instability. The performance of the TOC and NN controllers in the presence of flexible body modes should be evaluated. If the resulting vibration levels are determined to be excessive, then both strategies should be redesigned to incorporate the relevant modes. For instance, the switching strategies can be augmented to incorporate flexible body motion. In this approach, the resulting control algorithms would be defined by complicated multi-dimensional switching surfaces, requiring multiple actuator switchings to execute typical rest-to-rest maneuvers. Alternatively, the rigid body control system can be supplemented by a decoupled flexible body control system employing strain based actuators. This approach is currently being investigated under the National Center for Advanced Information Components Manufacturing (NCAICM) Phase II effort. For this project, lead zirconate titanate (PZT) stack actuators are being imbedded into the surface of the platen to suppress vibrational motion, thus permitting significant increases in the rigid body controller bandwidth. Although preliminary simulations are encouraging, no emperimental data is yet available. 


\subsection{CONCLUSIONS}

This report summarized an investigation into time-optimal control for the magnetically levitated photolithography platen. Since the nonlinearities associated with magnetic levitation actuators preclude the direct application of classical time-optimal control methodologies, two alternate approaches were developed using a computer simulation. In the first approach, conservative estimates of the available control forces were used to generate suboptimal switching curves that circumvent the system nonlinearities. In the second approach, exact solutions were determined iteratively and used as a training set for an artificial neural network. This solution approach has the added benefit that an accurate model of the system is not required. Instead, this approach can be applied directly to the hardware to generate a training set. The trained network provided optimal actuator switching times that incorporate the full nonlinear effects of the magnetic levitation actuators. Sample problems illustrated the effectiveness of these techniques as compared to traditional PD control. The TOC and NN controllers provided average reductions in settling times of 50\% and $70 \%$ over a wide range of motion. The neural network based control consistently provided the greatest improvement due to its full utilization of the available actuator forces and the precise switching provided by the open-loop clock. However, both controllers resulted in increased excitation of the out of plane motion as a result of lost compensation during the maneuver. The significance of this out of plane motion has not yet been determined. 


\subsection{REFERENCES}

1. Baz, A., Poh, S., Ro, J., Mutua, M., and Gilheany, J., "Active Control of NitinolReinforced Composite Beam," Intelligent Structural Systems, 1992, pp. 169-212.

2. Dosch, J.J., Leo, D.J., and Inman, D.J., "Comparison of Control Schemes for a Smart Antenna," Proceedings of the 31st Conference on Decision and Control, Tucson Arizona, December 1992, pp. 1815-1820.

3. Segalman, D.J., Witkowski, W.R., Adolf, D.B., and Shahinpoor, M., "Theory and Application of Electrically Controlled Polymeric Gels," Journal of Smart Materials and Structures, Vol. 1, 1992, pp. 95-100.

4. Trumper, D.L., Sanders, J.C., Nguyen, T.H., and Quenn, M.A., "Experimental Results in Nonlinear Compensation of a One-Degree-of-Freedom Magnetic Suspension," International Symposium on Magnetic Suspension Technology, NASA Langley Research Center, Hampton, VA, August 1991.

5. Arling, R.W., and Kohler, S.M., "Six Degree of Freedom Fine Motion Positioning Stage Based on Magnetic Levitation," presented at the NASA Conference on Magnetic Levitation, August 1993.

6. Redmond, J., "Nearly Time-Optimal Feedback Control of a Magnetically Levitated Photolithography Positioning System," Proceedings of the 1994 North American Conference on Smart Structures and Materials, February 1994.

7. Narendra, K.S. and Parthasarathy, K., "Identification and Control of Dynamical Systems Using Neural Networks," IEEE Transactions on Neural Networks, Vol. 1, No. 1, March 1991, pp. 4-27.

8. Swiniarski, R., "Neural Network Application to Adaptive Time-Optimal Control of Nonlinear Systems," Proceedings of the IEEE International Workshop on Intelligent Motion Control, Istanbul, Turkey, August 1990, pp.233-238.

9: Kohler, S.M., and Kahle, P.M., "Development of a Control System for the GCA Prototype Stage," Internal Report, Sandia National Labs, 1993.

10. O'Neil, P.V., Advanced Engineering Mathematics, Wadsworth, Inc., Belmont, CA, 1983.

11. Sage, A.P., White, C.C. III, Optimum Systems Control, Prentice Hall, Inc., Englewood Cliffs, NJ, Second Edition, 1977.

12. Athans, M., and Falb, P.L., Optimal Control, McGraw Hill Book Company, New York, NY, 1966.

13. Billingsley, J., "On the Design of Position Control Systems," IEE Proceedings-D, Vol. 138, No. 4, July 1991, pp. 331-336. 
14. Harvey, C.A., and Bugajski, D.J., "Smooth Blend of Time-Optimal and Linear Control," Journal of Guidance, Control, and Dynamics, Vol. 15, No. 2, March-April 1992, pp. 520-522.

15. Specht, D.F., "A General Regression Neural Network," IEEE Transactions on Neural Networks, Nov. 1991, pp. 568-576. 


\begin{tabular}{|c|c|c|}
\hline \multicolumn{3}{|c|}{ Distribution: } \\
\hline MS 0321 & 1400 & E.H. Barsis \\
\hline MS 1111 & 1421 & S.S. Dosanjh \\
\hline MS 1110 & 1422 & R.C. Allen, Jr. \\
\hline MS 1110 & 1423 & E.F. Brickell \\
\hline MS 1109 & 1424 & A.L. Hale \\
\hline MS 0439 & 1434 & J.L. Dohner \\
\hline MS 0439 & 1434 & D.R. Martinez \\
\hline MS 0439 & 1434 & G.G. Parker \\
\hline MS 0439 & 1434 & J.M. Redmond (3) \\
\hline MS 0439 & 1434 & D.J. Segalman \\
\hline MS 0841 & 1500 & D.J. McCloskey \\
\hline MS 0828 & 1502 & P.J. Hommert \\
\hline MS 0441 & 1503 & J.H. Biffle \\
\hline MS 0827 & 1511 & J.S. Rottler \\
\hline MS 0835 & 1513 & R.D. Skocypec \\
\hline MS 0443 & 1517 & H.S. Morgan \\
\hline MS 0439 & 1518 & R.K. Thomas \\
\hline MS 0501 & 2338 & S.M. Kohler (5) \\
\hline MS 0501 & 2338 & T.G. Smith \\
\hline MS 0501 & 2338 & S.L. Stanton \\
\hline MS 0501 & 2338 & S.D. Tucker (3) \\
\hline MS 0961 & 2403 & D.L. Plymale \\
\hline MS 0641 & 2643 & R.S. Urenda \\
\hline MS 0557 & 2741 & T.J. Baca \\
\hline MS 0557 & 2741 & J.J. Allen \\
\hline MS 0557 & 2741 & R. Rodeman \\
\hline MS 0557 & 2741 & P.S. Barney \\
\hline MS 0953 & 2900 & W.E. Alzheimer \\
\hline MS 0100 & $7613-2$ & Document Processing for DOE/OSTI (5) \\
\hline MS 9161 & 8342 & R.W.Arling \\
\hline MS 9018 & $8523-2$ & Central Technical Files \\
\hline MS 0899 & 13414 & Technical Library (5) \\
\hline MS 0619 & 13416 & Technical Publications \\
\hline
\end{tabular}

